\title{
An Algorithmic Framework for Labeling Network Maps`
}

\author{
Jan-Henrik Haunert ${ }^{1}$ and Benjamin Niedermann ${ }^{2}$ \\ 1 University of Osnabrück, Germany \\ 2 Karlsruhe Institute of Technology, Germany
}

\begin{abstract}
Drawing network maps automatically comprises two challenging steps, namely laying out the map and placing non-overlapping labels. In this paper we tackle the problem of labeling an already existing network map considering the application of metro maps. We present a flexible and versatile labeling model that subsumes different labeling styles. We show that labeling a single line of the network is NP-hard, even if we make very restricting assumptions about the labeling style that is used with this model. For a restricted variant of that model, we then introduce an efficient algorithm that optimally labels a single line with respect to a given cost function. Based on that algorithm, we present a general and sophisticated workflow for multiple metro lines, which is experimentally evaluated on real-world metro maps.
\end{abstract}

\section{Introduction}

Label placement and geographic network visualization are classical problems in cartography, which independently of each other have received the attention of computer scientists. Label placement usually deals with annotating point, line or area features of interest in a map with text labels such that the associations between the features and the labels are clear and the map is kept legible [8]. Geographic network visualization, on the other hand, often aims at a geometrically distorted representation of reality that allows information about connectivity, travel times, and required navigation actions to be retrieved easily. Computing a good network visualization is thus related to finding a layout of a graph with certain favorable properties [15]. For example, to avoid visual clutter in metro maps, an octilinear graph layout is often chosen, in which the orientation of each edge is a multiple of $45^{\circ}$ [11]12]14. Alternatively, one may choose a curvilinear graph layout, that is, to display the metro lines as curves [5]13.

Computing a graph layout for a metro map and labeling the stops have been considered as two different problems that can be solved in succession 14, but also integrated solutions have been suggested 1112. Nevertheless, in practice, metro maps are often drawn manually by cartographers or designers, as the existing algorithms do not achieve results of sufficient quality in adequate time. For example, Nöllenburg and Wolff [11] report that their method needed 10 hours and 31 minutes to compute a labeled metro map of Sydney that they present in their article, while an unlabeled map for the same instance was obtained after 23 minutes - both results were obtained without proof of optimality but with similar optimality gaps. On the other hand Wang and Chi [14 present an algorithm that creates the graph layout and labeling within one second, but they cannot guarantee that labels do not overlap each other or the metro lines.

An integrated approach to computing a graph layout and labeling the stops allows consideration to be given to all quality criteria of the final visualization. On the other hand, treating both problems separately will probably reduce computation time. Moreover, we consider the labeling of a metro map as an interesting problem on its own, since, in some situations, the layout of the network is given as part of the input and must not be changed. In a semi-automatic workflow, for example, a cartographer may want to draw or alter a graph layout manually before using an automatic method to place labels,

\footnotetext{
* A preliminary version of this paper has appeared in Proc. 21st Int. Conf. on Computing Combinatorics (COCOON'15), volume 9198 of Lect. Notes Comput. Sci., pages 689-700, Springer-Verlag. This research was initiated during Dagstuhl Seminar 13151 "Drawing Graphs and Maps with Curves" in April 2013
} 
probably to test multiple different labeling styles with the drawing. Hence, a labeling algorithm is needed that is rather flexible in dealing with different labeling styles.

In this paper, we are given the layout of a metro map consisting of several metro lines on which stops (also called stations) are located. For each stop we are further given its name, which should be placed close to its position. We first introduce a versatile and general model for labeling metro maps; see Section 2. Like many labeling algorithms for point sets [136], our algorithm uses a discrete set of candidate labels for each point. Often, each label is represented by a rectangle wrapping the text. Since we also want to use curved labels, however, we represent a label by a simple polygon that approximates a fat curve, that is, a curve of certain width reflecting the text height. We then prove that even in that simple model labeling a single metro line is NP-hard considering different labeling styles. Hence, we restrict the set of candidates satisfying certain properties, which allows us to solve the problem on one metro line $C$ in $O\left(n^{2}\right)$ time, where $n$ is the number of stops of $C$; see Section 4 . This algorithm optimizes the labeling with respect to a cost function that is based on Imhof's [8] classical criteria of cartographic quality. Utilizing that algorithm, we present an efficient heuristic for labeling a metro map consisting of multiple metro lines; see Section 6. Our method is similar to the heuristic presented by Kakoulis and Tollis [9, in the sense that it discards some label candidates to establish a set of preconditions that allow for an efficient exact solution. Our model of quality is more general than the one of Kakoulis and Tollis, however, as it not only takes the quality of individual labels but also the quality of pairs of labels for consecutive metro stations into account. Finally, we evaluate our approach presenting experiments conducted on realistic metro maps; see Section 8 8 . Note that "stops" on "metro lines" can refer more generally to points of interest on the lines of any kind of a network map. We address labeling styles for octilinear graph layouts and curvilinear graph layouts that use Bézier curves. The more general model behind our method, however, subsumes but is not limited to these particular styles.

\section{Labeling Model}

We assume that the metro lines are given by directed, non-self-intersecting curves in the plane described by polylines, which for example have been derived by approximating Bézier curves. We denote that set of metro lines by $\mathcal{M}$. Further, the stops of each metro line $C \in \mathcal{M}$ are given by an ordered set $\mathcal{S}_{C}$ of points on $C$ going from the beginning to the end of $C$. For two stops $s, s^{\prime} \in \mathcal{S}_{C}$ we write $s<s^{\prime}$ if $s$ lies before $s^{\prime}$. We denote the union of the stops among all metro lines by $\mathcal{S}$ and call the pair $(\mathcal{M}, \mathcal{S})$ a metro map.

For each stop $s \in \mathcal{S}$ we are further given a name that should be placed close to it. In contrast to previous work, we do not follow traditional map labeling abstracting from the given text by bounding boxes. Instead we model a label $\ell$ of a stop $s \in \mathcal{S}$ as a simple polygon. For example, a label could have been derived by approximating a fat curve prescribing the name of the stop; see Fig. 1. For each stop $s$ we are given a set $\mathcal{K}_{s}$ of labels, which we also call candidates of $s$. The set $\bigcup_{s \in \mathcal{S}} \mathcal{K}_{s}$ is denoted by $\mathcal{K}$.

Since "names should disturb other map content as little as possible" [8, we strictly forbid overlaps between labels and lines as well as label-label overlaps. Further, each stop must be labeled. Hence, a set $\mathcal{L} \subseteq \mathcal{K}$ is called a labeling if (1) no two labels of $\mathcal{L}$ intersect each other, (2) no label $\ell \in \mathcal{L}$ intersects any metro line $C \in \mathcal{M}$, and (3) for each stop $s \in \mathcal{S}$ there is exactly one label $\ell \in \mathcal{L} \cap \mathcal{K}_{s}$.

\section{Definition 1 (MetroMapLabeling).}

Given: Metro map $(\mathcal{M}, \mathcal{S})$, candidates $\mathcal{K}$ and cost function $w: 2^{\mathcal{K}} \rightarrow \mathbb{R}^{+}$.

Find, if it exists: Optimal labeling $\mathcal{L}$ of $(\mathcal{M}, \mathcal{S}, \mathcal{K}, w)$, i.e., $w(\mathcal{L}) \leq w\left(\mathcal{L}^{\prime}\right)$ for any labeling $\mathcal{L}^{\prime} \subseteq \mathcal{K}$.

The model allows us to create arbitrarily shaped label candidates for a metro map. In our evaluation we have considered two different labeling styles. The first style, OCtiLinStyle, creates for each stop a set of octilinear rectangles as label candidates; see Fig. 2. We use that style for octilinear maps. The second style, CURVEDStYle, creates for each stop a set of fat Bézier curves as label candidates, which are then approximated by simple polygons; see Fig. 1. We use that style for curvilinear metro maps, 


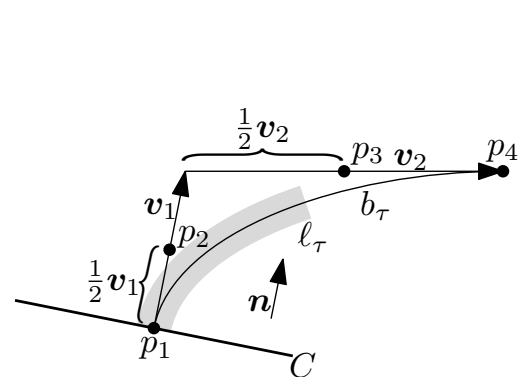

(a)

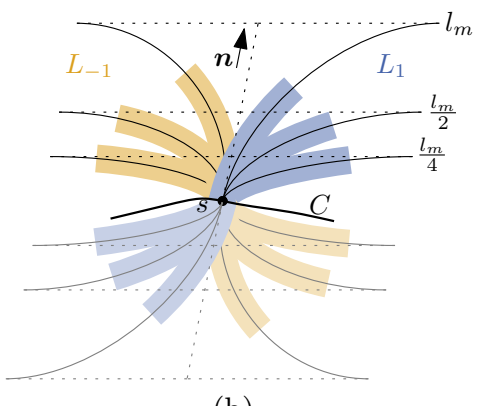

(b)

Fig. 1: Construction of curved candidates. (a) Construction of a single label. (b) Candidates $\mathcal{K}_{s}=$ $L_{1} \cup L_{-1}$ for stop $s$.

in order to adapt the curvilinear style of the metro map. The basic idea is that a label perpendicularly emanates from a stop with respect to its metro line and then becomes horizontal to sustain legibility. In the following section we motivate our choice of candidates based on cartographic criteria and give detailed technical descriptions for both labeling styles.

\subsection{Two Examples of Labeling Styles}

We extracted the rules for generating label candidates from Imhof's "general principles and requirements" for map labeling $[8$. For schematic network maps, the need for "legibility" implies that we must not destroy the underlying design principle with clutter. To this end, we generate candidate labels that adhere to the schematics of the network. That is, we use straight horizontal and diagonal labels with octilinear layouts and curved labels with curvilinear layouts.

We now describe more precisely, how we defined the labeling styles CURVEDStYle and OCtiLinSTYLE, which are used for curvilinear layouts and octilinear layouts, respectively.

Curvilinear Metro Maps. For CurvedStyle, assume that the given metro map is curvilinear. In order to achieve a "clear graphic association" between a label and the corresponding point $p$, we construct the simple polygon prescribing a candidate label based on a curve $\ell$ (possibly a straight-line segment) that emanates from $p$. The candidate label itself is a continuous section of $\ell$ that does not directly start in $p$ but at a certain configurable distance from it. We define the end of the candidate label on $\ell$ based on the text length and assign a non-zero width to the curve section to represent the text height. In the case that $p$ lies on a single curved line $C$, we require that $\ell$ and $C$ are perpendicular in $p$ to enhance the angular resolution of the final drawing. By bending $\ell$ towards the horizontal direction, we avoid steep labels. We approximate $\ell$ by a simple polygon consisting of a constant number of line segments.

We now describe the construction of a single candidate more specifically. For each stop $s$ of each metro line $C$ we create a constant number of curved labels adapting the curvilinear style of the metro map. The basic idea is that a label perpendicularly emanates from $s$ with respect to $C$ and then becomes horizontal to sustain legibility; see Fig. 1. Let $\boldsymbol{n}=\left(n_{x}, n_{y}\right)$ be the normalized normal vector of $C$ at $s$. Further, let $d \in\{-1,1\}$ and $c_{1}, c_{2} \in \mathbb{R}^{+}$be pre-defined constants. For $\tau=\left(c_{1}, c_{2}, d\right)$ we define the fat cubic Bézier curve $b_{\tau}$ by the following four control points; see Fig. 1. $p_{1}=s, p_{2}=$ $s+0.5 \cdot \boldsymbol{v}_{1}, p_{3}=s+\boldsymbol{v}_{1}+0.5 \cdot \boldsymbol{v}_{2}, p_{4}=s+\boldsymbol{v}_{1}+\boldsymbol{v}_{2}$, where $\boldsymbol{v}_{1}=c_{1} \cdot \boldsymbol{n}, \boldsymbol{v}_{2}=\operatorname{sgn}(\boldsymbol{n}) \cdot\left(d \cdot c_{2}, 0\right)$, and $\operatorname{sgn}(\boldsymbol{n})=1$ if $n_{x}>0$ and $\operatorname{sgn}(\boldsymbol{n})=-1$ otherwise. We define the thickness of $b_{\tau}$ to be the predefined height of a label. Let $\ell_{\tau}$ be the sub-curve of $b_{\tau}$ that starts at $p_{1}$ and has the length of the name of $s$ and let $\ell_{\tau}^{\prime}$ be the curve when mirroring $\ell_{\tau}$ at $s$. Further, let $l_{m}$ be the length of the longest name of a stop in $\mathcal{S}$ and let $L_{d}=\left\{\ell_{\tau}, \ell_{\tau}^{\prime} \mid \tau \in\left\{\left(l_{m}, l_{m}, d\right),\left(\frac{l_{m}}{2}, l_{m}, d\right),\left(\frac{l_{m}}{4}, l_{m}, d\right)\right\}\right\}$. If $\boldsymbol{n}$ has an orientation less than or equal to $60^{\circ}$, we set $\mathcal{K}_{s}=L_{1}$ and otherwise $\mathcal{K}_{s}=L_{1} \cup L_{-1}$. Hence, if $\boldsymbol{n}$ is almost vertical and $C$ 


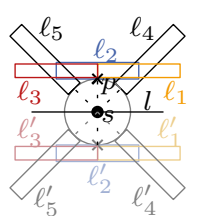

(a)

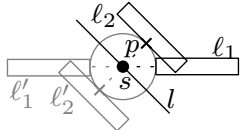

(b)

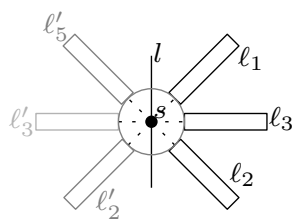

(c)

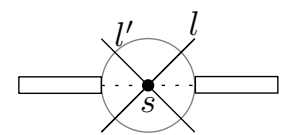

(d)

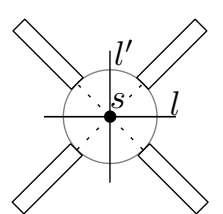

(e)

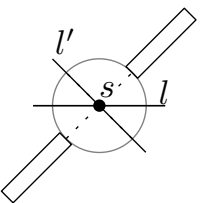

(f)

Fig. 2: Construction of octilinear candidates for a stop $s .(\mathrm{a}) \mathrm{s}$ lies on a horizontal segment. (b) $s$ lies on a diagonal segment. (c) $s$ lies on a vertical segment. (d) $s$ lies on a crossing of two diagonal segments $l$ and $l^{\prime}$. (e) $s$ lies on a crossing of a vertical and horizontal segment. (f) $s$ lies on a crossing of a vertical and diagonal segment.

is therefore almost horizontal at $s$, we also add the labels $L_{-1}$ pointing into the opposite $x$-direction than $\boldsymbol{n}$. In our experiments we did not let the labels start at $s$, but with a certain offset to $s$, in order to avoid intersections with $C$.

Octilinear Metro Maps. For OctilinStyle assume that the metro map is octilinear. We model the labels as horizontal and diagonal rectangles. Let $l$ be the line segment of $C$ on which $s$ lies and let $R$ be an axis-aligned rectangle that is the bounding box of the name of $s$. Further, let $c$ be a circle around $s$ with a pre-defined radius. We place the labels such that they touch the border of $c$, but they do not intersect the interior of $c$. Hence, the labels have a pre-defined offset to $s$.

If $l$ is horizontal, we place five copies $\ell_{1}, \ldots, \ell_{5}$ of $R$ above $l$ as follows; see Fig. 2(a). We place $\ell_{1}, \ell_{2}$ and $\ell_{3}$ such that the left-bottom corner of $\ell_{1}$, the midpoint of $\ell_{2}$ 's bottom edge and the right-bottom corner of $\ell_{3}$ coincides with the topmost point of $c$. We rotate $\ell_{4}$ by $45^{\circ}$ counterclockwise and place it at $c$ such that the midpoint of its left side touches $c$, i.e., that midpoint lies on a diagonal through $s$. Finally, $\ell_{5}$ is obtained by mirroring $\ell_{4}$ at the vertical line through $s$. Mirroring $\ell_{1}, \ldots, \ell_{5}$ at the horizontal line through $s$, we obtain the rectangles $\ell_{1}^{\prime}, \ldots, \ell_{5}^{\prime}$, respectively. We then set $\mathcal{K}_{s}=\left\{\ell_{i}, \ell_{i}^{\prime} \mid 1 \leq i \leq 5\right\}$.

If $l$ is diagonal, we create the candidates in the same manner as in the case that $l$ is horizontal; see Fig. 2(b). However, we only create the candidates $\ell_{2}$ and $\ell_{2}^{\prime}$ and the candidates that are horizontally aligned.

If $l$ is vertical, we place three copies $\ell_{1}, \ldots, \ell_{3}$ of $R$ to the right of $l$ as follows; see Fig. 2(c). We rotate $\ell_{1}$ by $45^{\circ}$ counterclockwise and $\ell_{2}$ by $45^{\circ}$ clockwise. We place $\ell_{1}, \ell_{2}, \ell_{3}$ at $c$ such that the midpoints of their left edges touch $c$. Mirroring $\ell_{1}, \ell_{2}, \ell_{3}$ at the vertical line through $s$, defines the rectangles $\ell_{1}^{\prime}, \ell_{2}^{\prime}$ and $\ell_{3}^{\prime}$. We set $\mathcal{K}_{s}=\left\{\ell_{i}, \ell_{i}^{\prime} \mid 1 \leq i \leq 3\right\}$.

In case that $s$ is a crossing of two metro lines, we create the candidates differently. If $s$ is the crossing of two diagonals, we create the candidates as shown in Fig. 2(d). If $s$ is the crossing of a horizontal and a vertical segment, we create the labels as shown in Fig. 2(e)] If $s$ is the crossing of a diagonal and horizontal segment, we create the labels as shown in Fig. 2(f)] We analogously create the labels, if $s$ is the crossing of a vertical and a diagonal segment.

Remark: If a stop lies on multiple metro lines, then we can apply similar constructions, where the labels are placed on the angle bisectors of the crossing lines.

\section{Computational Complexity}

We first study the computational complexity of METROMAPLABELING assuming that the labels are either based on OCTILINSTYLE or CURVEDSTYLE. In particular we show that the problem is NP-hard, if the metro map consists of only one line. The proof uses a reduction from the NP-complete problem monotone planar 3SAT [10]. Based on the given style, we create for the set $\mathcal{C}$ of 3SAT clauses a metro map $(\mathcal{M}, \mathcal{S})$ such that $(\mathcal{M}, \mathcal{S})$ has a labeling if and only if $\mathcal{C}$ is satisfiable. The proof can be easily adapted to other labeling styles. Note that the complexity of labeling points using a finite set of axisaligned rectangular label candidates is a well-studied NP-complete problem, e.g., see [76]. However, 


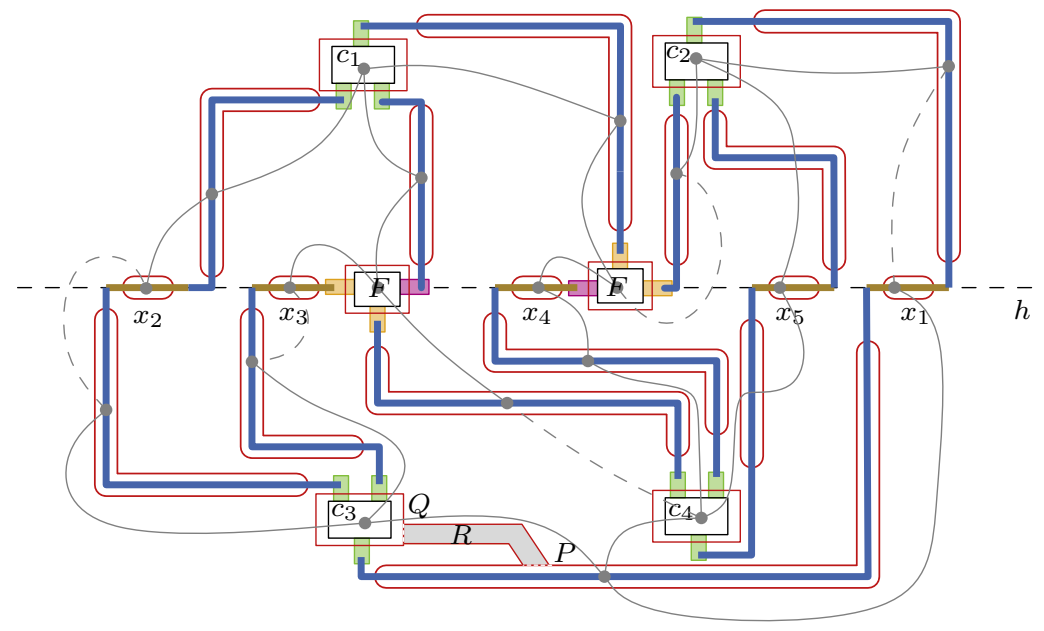

Fig. 3: Illustration of NP-completeness proof. (a) 3-SAT formula $\varphi$ with clauses $c_{1}=x_{4} \vee x_{1} \vee x_{5}$, $c_{2}=x_{2} \vee x_{4} \vee x_{3}, c_{3}=\bar{x}_{2} \vee \bar{x}_{1} \vee \bar{x}_{3}$ and $c_{4}=\bar{x}_{3} \vee \bar{x}_{5} \vee \bar{x}_{4}$ represented as a metro map. Truth assignment is $x_{1}=$ true, $x_{2}=$ true, $x_{3}=$ false, $x_{4}=$ false and $x_{5}=$ false. The gray graph $G$ represents the adjacencies of the used gadgets. The solid lines represent a spanning tree of $G$ that can be used to merge the polygons to one simple polygon. Exemplarily the polygons $P$ and $Q$ are merged into one polygon $R$. The single components are illustrated in Fig. 4 .

since we do not necessarily use axis-aligned rectangles as labels and since for the considered labeling styles the labels are placed along metro lines, it is not obvious how to reduce a point-feature labeling instance on an instance of MetromapLabeling. In order to show the NP-hardness, we prove that it is NP-complete to decide whether a metro map $(\mathcal{M}, \mathcal{S})$ has a labeling based on the given labeling style.

Theorem 1. Metromaplabeling is NP-complete, if the labels are based on OctilinStYle or CurvedSTYLE, even if the metro map has only one metro line.

Proof. For the illustrations we use OctilinStyle, but the same constructions can be done based on CurvedStyle; see end of proof. We first show that the problem deciding whether $(\mathcal{M}, \mathcal{S})$ has a labeling lies in NP. We first create for each stop $s \in \mathcal{S}$ its candidates $\mathcal{K}_{s}$ based on the given labeling style. Recall that each candidate has constant size. We then guess for each stop $s \in \mathcal{S}$ the label $\ell_{s}$ that belongs to the desired labeling $\mathcal{L}$. Obviously, we can decide in polynomial time whether $\left\{\ell_{s} \mid s \in \mathcal{S}\right\}$ is a labeling of $(\mathcal{M}, \mathcal{S})$ performing basically intersection tests.

We now perform a reduction from the NP-complete Planar Monotone 3-SaT problem [10. Let $\varphi$ be a Boolean formula in conjunctive normal form such that it consists of $n$ variables and $m$ clauses and, furthermore, each clause contains at most three literals. The formula $\varphi$ induces the graph $G_{\varphi}$ as follows. $G_{\varphi}$ contains for each variable a vertex and it contains for each clause a vertex. Two vertices $u$ and $v$ are connected by an edge $\{u, v\} \in E$ if and only if $u$ represents a variable $x$ and $v$ represents a clause $c$, such that $x$ is contained in $c$. We call a clause of $\varphi$ positive (negative) if it contains only positive (negative) literals.

The formula $\varphi$ is an instance of Planar Monotone 3-SAT if it satisfies the following requirements.

1. $\varphi$ is monotone, i.e., each clause is either positive or negative, and

2. the graph $G_{\varphi}$ is planar and has a rectilinear plane embedding such that

(a) the vertices representing variables are placed on a horizontal line $h$,

(b) the vertices representing negative clauses are placed below $h$,

(c) the vertices representing positive clauses are placed above $h$, 
(a)

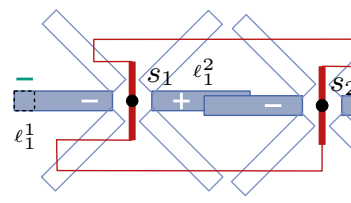

(c)

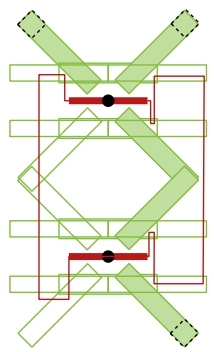

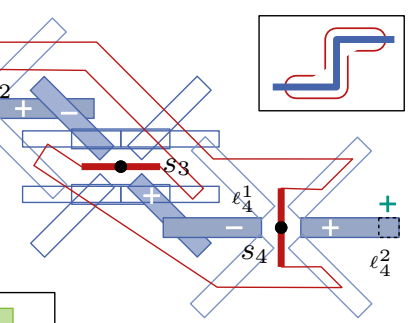

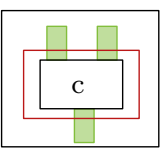

(d)

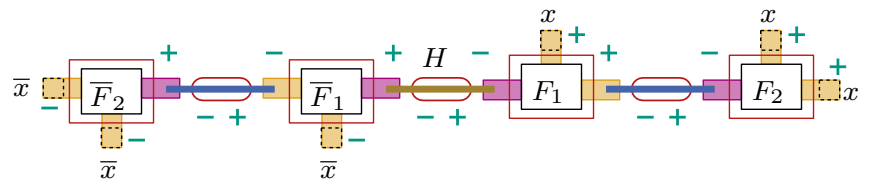

Fig. 4: Illustration of the gadgets. Selectable labels are filled, while all other labels are not filled. Ports are marked with a dashed square. (a) Chain gadget of length 4. (b) Fork gadget. (c) Clause gadget. (d) Variable gadget with three negative and three positive ports.

(d) the edges are drawn on their respective side of $h$.

Planar Monotone 3-SAT then asks whether $\varphi$ is satisfiable.

Using only stops lying on single horizontal and vertical segments, we construct a metro map $(\mathcal{M}, \mathcal{S})$ that mimics the embedding of $G_{\varphi}$. In particular $\mathcal{M}$ will only consist of one metro line $C$ that connects all stops such that the stops and their candidates simulate the variables and clauses of $\varphi$. We will prove that $(\mathcal{M}, \mathcal{S})$ has a labeling if and only if $\varphi$ is satisfiable. We refer to Fig. 3 for a sketch of the construction. We first define gadgets simulating variables, clauses and connecting structures. Each gadget consists of a set of stops that lie on the border of a simple polygon $P$. Later on we use this polygon $P$ to prescribe the shape of the metro line $C$.

Chain. The chain gadget represents and transmits truth values from variables to clauses mimicking the embeddings of the edges in $G_{\varphi}$. A chain consists of an even number of stops $s_{1}, \ldots, s_{k}$ that lie on vertical and horizontal segments; see Fig. 4(a). Hence, with respect to the given labeling style each stop $s_{i}$ has a predefined set of candidates $\mathcal{K}_{s_{i}}$. For each stop $s_{i}$ there are two specially marked candidates $\ell_{i}^{1}$ and $\ell_{i}^{2}$ that lie on opposite sides of $s_{i}$ 's segment; for an example see the filled blue labels in Fig 4(a). We say that those labels are selectable, because we define the gadget such that those labels are the only labels that can be selected for a labeling. To that end, we lay out the metro line such that it does not intersect any selectable label, but all labels that are not selectable. The stops are placed such that the following conditions are satisfied.

(1) The label $\ell_{i}^{2}$ intersects the label $\ell_{i+1}^{1}$ for $1 \leq i<k$.

(2) Except the intersections mentioned in (1), there is no intersection between selectable labels of different stops.

(3) The segments of the stops are connected by polylines s.t. the result is a simple polygon $P$ intersecting all labels except the selectable labels.

The labels $\ell_{1}^{1}$ and $\ell_{k}^{2}$ do not intersect any selectable labels; we call them the ports of the chain. Later on, we use the ports to connect other gadgets with the chain, i.e., we arrange the gadgets such that two of their ports intersect, but no other selectable label. Further, we assign a polarization to each selectable label. The labels $\ell_{1}^{1}, \ldots, \ell_{k}^{1}$ are negative and the labels $\ell_{1}^{2}, \ldots, \ell_{k}^{2}$ are positive.

Consider a labeling $\mathcal{L}$ of a chain assuming that $P$ is interpreted as a metro line; we can cut $P$ at some point in order to obtain an open curve. By construction of $P$ only selectable labels are contained in $\mathcal{L}$. In particular we observe that if the negative port $\ell_{1}^{1}$ is not contained in $\mathcal{L}$, then the positive labels $\ell_{1}^{2}, \ldots, \ell_{k}^{2}$ belong to $\mathcal{L}$. Analogously, if the positive port $\ell_{k}^{2}$ is not contained in $\mathcal{L}$, then the 
negative labels $\ell_{1}^{1}, \ldots, \ell_{k}^{1}$ belong to $\mathcal{L}$. We use this behavior to represent and transmit truth values through the chain.

Fork. The fork gadget splits an incoming chain into two outgoing chains and transmits the truth value represented by the incoming chain into the two outgoing chains. A fork consists of three stops $s_{1}$, $s_{2}$ and $s_{3}$ such that $s_{1}$ and $s_{2}$ are placed on vertical segments and $s_{3}$ is placed on a horizontal segment; see Fig. $4(\mathrm{~b})$. Analogously to the chain, each stop $s_{i}$ with $(1 \leq i \leq 3)$ has two selectable labels $\ell_{i}^{1}$ and $\ell_{i}^{2}$. We arrange the stops such that the following conditions are satisfied.

1. The labels $\ell_{2}^{1}$ and $\ell_{3}^{1}$ intersect $\ell_{1}^{2}$. Apart from those two intersections no selectable label intersects any other selectable label.

2. The segments of the stops are connected by polylines s.t. the result is a simple polygon $P$ intersecting all labels except the selectable labels.

The label $\ell_{1}^{1}$ is the incoming port and the labels $\ell_{2}^{2}$ and $\ell_{3}^{2}$ are the outgoing ports of the fork. We distinguish two types of forks by assigning different polarizations to the selectable labels. In the negative (positive) fork, the labels $\ell_{1}^{1}, \ell_{2}^{1}$ and $\ell_{3}^{1}$ are positive (negative) and the labels are $\ell_{1}^{2}, \ell_{2}^{2}$ and $\ell_{3}^{2}$ are negative (positive). Hence, the incoming port is positive (negative) and the outgoings ports are negative (positive).

Consider a labeling $\mathcal{L}$ of a fork assuming that $P$ is interpreted as a metro line. By construction of $P$ only selectable labels belong to $\mathcal{L}$. Further, if the incoming port $\ell_{1}^{1}$ does not belong to $\mathcal{L}$, then the outgoing ports $\ell_{1}^{2}$ and $\ell_{1}^{2}$ belong to $\mathcal{L}$. Finally, if one outgoing port does not belong to $\mathcal{L}$, then the incoming port belongs to $\mathcal{L}$.

Clause. The clause gadget represents a clause $c$ of the given instance. It forms a chain of length 2 with the addition that it has three ports instead of two ports; see Fig 4 (c). To that end one of both stops has three selectable labels; one intersecting a selectable label of the other stop, and two lying on the opposite side of the stop's segment without intersecting any selectable label of the other stop. The gadget is placed at the position where the vertex of $c$ is located in the drawing of $G_{\varphi}$; see Fig. 3 . We observe that a labeling $\mathcal{L}$ of a clause gadget always contains at least one port. Further, we do not assign any polarization to its selectable labels.

Variable. The variable gadget represents a single variable $x$. It forms a composition of chains and forks that are connected by their ports; see Fig 4(d). More precisely, let $s$ be the number of clauses in which the negative literal $\bar{x}$ occurs and let $t$ be the number of clauses in which the positive literal $x$ occurs. Along the horizontal line $h$ on which the vertex of $x$ is placed in the drawing of $G_{\varphi}$, we place a horizontal chain $H$. Further, we place a sequence of negative forks $\bar{F}_{1}, \ldots, \bar{F}_{s-1}$ to the left of $H$ and a sequence of positive forks $F_{1}, \ldots, F_{t-1}$ to the right of $H$. The negative incoming port of $F_{1}$ is connected to the positive port of $H$ by a chain. Two consecutive forks $F_{i}$ and $F_{i+1}$ are connected by a chain $H^{\prime}$ such that $H^{\prime}$ connects a positive outgoing port of $F_{i}$ with the negative incoming port of $F_{i+1}$. Analogously, the positive incoming port of $\bar{F}_{1}$ is connected to the negative port of $H$ by a chain. Two consecutive forks $\bar{F}_{i}$ and $\bar{F}_{i+1}$ are connected by a chain $H^{\prime}$ such that $H^{\prime}$ connects a negative outgoing port of $\bar{F}_{i}$ with the positive incoming port of $\bar{F}_{i+1}$.

We observe that the gadget has $s+t$ free ports. Further, we can arrange the forks such that the free ports of $F_{1}, \ldots, F_{t-1}$ lie above $h$ and the free ports of $\bar{F}_{1}, \ldots, \bar{F}_{s-1}$ lie below $h$.

Consider a labeling $\mathcal{L}$ of a variable. By construction of the forks and chains, if one positive free port is not contained in $\mathcal{L}$, then all negative free ports must be contained in $\mathcal{L}$. Analogously, if one negative free port is not contained in $\mathcal{L}$, then all positive free ports must be contained in $\mathcal{L}$.

Using additional chains we connect the positive free ports with the positive clauses and the negative ports with the negative clauses correspondingly; see Fig. 3. More precisely, assume that the variable $x$ is contained in the positive clause $c$; negative clauses can be handled analogously. With respect to the drawing of $G_{\varphi}$, a positive free port of $x$ 's gadget is connected with the negative port of a chain whose positive port is connected with a free port of c's gadget. Note that we can easily choose the simple polygons enclosing the gadgets such that they do not intersect by defining them such that they surround the gadgets tightly. 
(a)
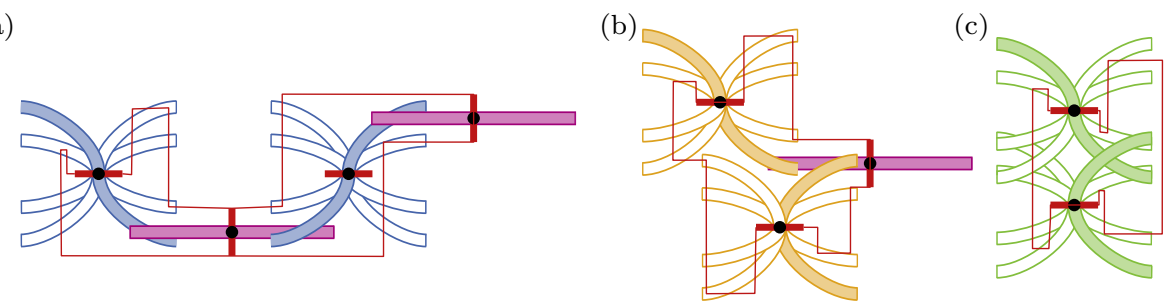

Fig. 5: Illustration of the gadgets based on OCTILINSTYLE. Selectable labels are filled, while all other labels are not filled. (a) Chain gadget of length 4. (b) Fork gadget. (c) Clause gadget.

One Metro Line. We construct the polygons enclosing the single gadgets such that they do not intersect each other. We now sketch how the polygons can be merged to a single simple polygon $P$. Cutting this polygon at some point we obtain a polyline prescribing the desired metro line.

We construct a graph $H=(V, E)$ as follows. The polygons of the gadgets are the vertices of the graph and an edge $(P, Q)$ is contained in $E$ if and only if the corresponding gadgets of the polygons $P$ and $Q$ are connected by their ports; see Fig. 3 . Since $G_{\varphi}$ is planar and the gadgets mimic the embedding of $G_{\varphi}$, it is not hard to see that $H$ is also planar. We construct a spanning tree $T$ of $H$. If an edge $(P, Q)$ of $H$ is also contained in $T$, we merge $P$ and $Q$ obtaining a new simple polygon $R$; see for an example Fig. 3. To that end we cut $P$ and $Q$ in polylines and connect the four end points by two new polylines such that the result is a simple polygon. We in particular ensure that the new polygon does not intersect any other polygon and that $R$ intersects the same labels as $P$ and $Q$ together. In $T$ we correspondingly contract the edge. Note that by contracting edges, $T$ remains a tree. We repeat that procedure until $T$ consists of a single vertex, i.e., only one simple polygon is left.

Soundness. It is not hard to see that our construction is polynomial in the size of the given 3SAT formula $\varphi$.

Assume that $\varphi$ is satisfiable. We show how to construct a labeling $\mathcal{L}$ of the constructed metro map. For each variable $x$ that is true (false) in the given truth assignment, we put all negative (positive) labels of the corresponding variable gadget and its connected chains into $\mathcal{L}$. By construction those labels do not intersect. It remains to select labels for the clause gadgets. Consider a positive clause $c$; negative clauses can be handled analogously. Since $\varphi$ is satisfiable, $c$ contains a variable $x$ that is true in the given truth assignment of $\varphi$. The set $\mathcal{L}$ contains only negative labels of the chain connecting the gadget of $x$ with the gadget of $c$, but no positive labels of that chain. Hence, we can add the port of $c$ 's gadget that is connected to that chain without creating intersections. For the second stop of the clause we put that selectable label into $\mathcal{L}$ that is not a port. We can apply this procedure to all positive and negative clauses without creating intersections, which yields the labeling $\mathcal{L}$ of the constructed metro map.

Finally, assume that we are given a labeling $\mathcal{L}$ of the constructed metro map. Consider the clause gadget of a positive clause $c$; negative clauses can be handled analogously. By construction $\mathcal{L}$ contains at least one port $\ell$ of that gadget. This port is connected to a chain, which is then connected to a gadget of a variable $x$. We set that variable $x$ true. We apply this procedure to all clauses; for negative clauses we set the corresponding variable to false. Since $\ell$ is contained in $\mathcal{L}$, only negative labels of that chain can be contained in $\mathcal{L}$, but no positive labels. Hence, the positive ports of the variable gadget are also not contained in $\mathcal{L}$. By the previous reasoning this implies that all negative ports of the gadget are contained in $\mathcal{L}$. Consequently, by applying a similar procedure to negative clauses, it cannot happen that $x$ is set to false. Altogether, this implies a valid truth assignment of $\varphi$.

Remarks: Fig. 5 illustrates the construction of the gadgets for CuRvEDSTYLE. Note that only the fork gadget, the clause gadget and the chain gadget rely on the concrete labeling style. Further, using CuRvedStyle, a stop $s$ lying on a vertical segment $l_{v}$ has exactly two different distinguish labels; one that lies to the left of $l_{v}$ and one that lies to the right of $l_{v}$. 

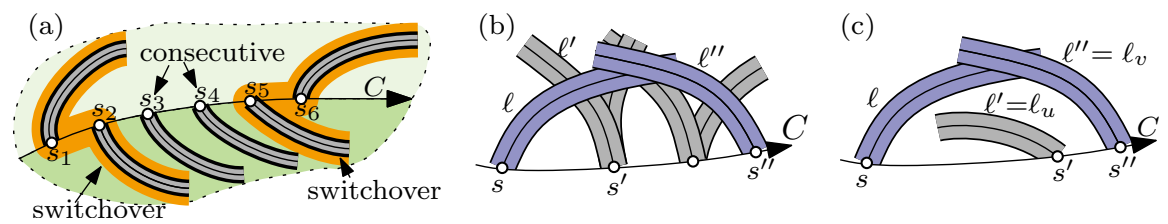

Fig. 6: (a) Consecutive stops and switchovers. (b) The candidates satisfy the transitivity property. (c) The candidates do not satisfy the transitivity property.

\section{Labeling Algorithm for a Single Metro Line}

We now study the case that the given instance $I=(\mathcal{M}, \mathcal{S}, \mathcal{K}, w)$ consists only of one metro line $C$. Based on cartographic criteria we introduce three additional assumptions on $I$, which allows us to efficiently solve MetromapLaBeling.

For each stop $s \in S$, we assume that each candidate $\ell \in \mathcal{K}_{s}$ is assigned to one side of $C$; either $\ell$ is a left candidate assigned to the left side of $C$, or $\ell$ is a right candidate assigned to the right side of $C$. For appropriately defined candidate sets those assignments correspond with the geometric positions of the candidates, i.e., left (right) candidates lie on the left (right) hand side of $C$.

Assumption 1 (Separated Labels) Candidates that are assigned to different sides of $C$ do not intersect.

This assumption is normally not a real restriction, because for appropriately defined candidate sets and realistic metro lines, the line $C$ separates both types of candidates geometrically. We further require what we call the transitivity property.

Assumption 2 (Transitivity Property) For any three stops $s, s^{\prime}, s^{\prime \prime} \in \mathcal{S}$ with $s<s^{\prime}<s^{\prime \prime}$ and any three candidates $\ell \in \mathcal{K}_{s}, \ell^{\prime} \in \mathcal{K}_{s^{\prime}}$ and $\ell^{\prime \prime} \in \mathcal{K}_{s^{\prime \prime}}$ assigned to the same side of $C$, it holds that if neither $\ell$ and $\ell^{\prime}$ intersect nor $\ell^{\prime}$ and $\ell^{\prime \prime}$ intersect then also $\ell$ and $\ell^{\prime \prime}$ do not intersect; see also Fig. G(b)-(c).

In our experiments we established Assumption 1 and Assumption 2 by removing candidates greedily. In Section 8 we show that for real-world metro maps and the considered candidate sets we remove only few labels, which indicates that those assumptions have only a little influence on the labelings.

Two stops $s, s^{\prime} \in \mathcal{S}$ with $s<s^{\prime}$ are consecutive if there is no other stop $s^{\prime \prime} \in \mathcal{S}$ with $s<s^{\prime \prime}<s^{\prime}$; see Fig. 6(a). For two consecutive stops $s_{1}, s_{2} \in \mathcal{S}$ we say that each two candidates $\ell_{1} \in \mathcal{K}_{s_{1}}$ and $\ell_{2} \in \mathcal{K}_{s_{2}}$ are consecutive and denote the set that contains each pair of consecutive labels in $\mathcal{L} \subseteq \mathcal{K}$ by $P_{\mathcal{L}} \subseteq \mathcal{L} \times \mathcal{L}$. Further, two consecutive labels $\ell_{1}, \ell_{2} \in \mathcal{K}_{C}$ form a switchover $\left(\ell_{1}, \ell_{2}\right)$ if they are assigned to opposite sides of $C$, where $\left(\ell_{1}, \ell_{2}\right)$ denotes an ordered set indicating the order of the stops of $\ell_{1}$ and $\ell_{2}$. Two switchovers of $C$ are consecutive in $\mathcal{L} \subseteq \mathcal{K}$ if there is no switchover in $\mathcal{L}$ in between of both. We define the set of all switchovers in $\mathcal{K}$ by $\mathcal{W}$ and the set of consecutive switchovers in $\mathcal{L} \subseteq \mathcal{K}$ by $\Gamma_{\mathcal{L}} \subseteq \mathcal{W} \times \mathcal{W}$.

Based on cartographic criteria extracted from Imhof's "general principles and requirements" for map labeling [8], we require a cost function $w: 2^{\mathcal{K}} \rightarrow \mathbb{R}^{+}$of the following form; see also Section 5 for a detailed motivation of $w$.

Assumption 3 (Linear Cost Function) For any $\mathcal{L} \subseteq K$ we require

$$
w(\mathcal{L})=\sum_{\ell \in \mathcal{L}} w_{1}(\ell)+\sum_{\left(\ell_{1}, \ell_{2}\right) \in P_{\mathcal{L}}} w_{2}\left(\ell_{1}, \ell_{2}\right)+\sum_{\left(\sigma_{1}, \sigma_{2}\right) \in \Gamma_{\mathcal{L}}} w_{3}\left(\sigma_{1}, \sigma_{2}\right),
$$

where $w_{1}: \mathcal{L} \rightarrow \mathbb{R}$ rates a single label, $w_{2}: P_{\mathcal{L}} \rightarrow \mathbb{R}$ rates two consecutive labels and $w_{3}: \Gamma_{\mathcal{L}} \rightarrow \mathbb{R}$ rates two consecutive switchovers.

In particular, we define $w$ such that it penalizes the following structures to sustain readbility. (1) Steep or highly curved labels. (2) Consecutive labels that lie on different sides of $C$, or that are shaped differently. (3) Consecutive switchovers that are placed close to each other. 


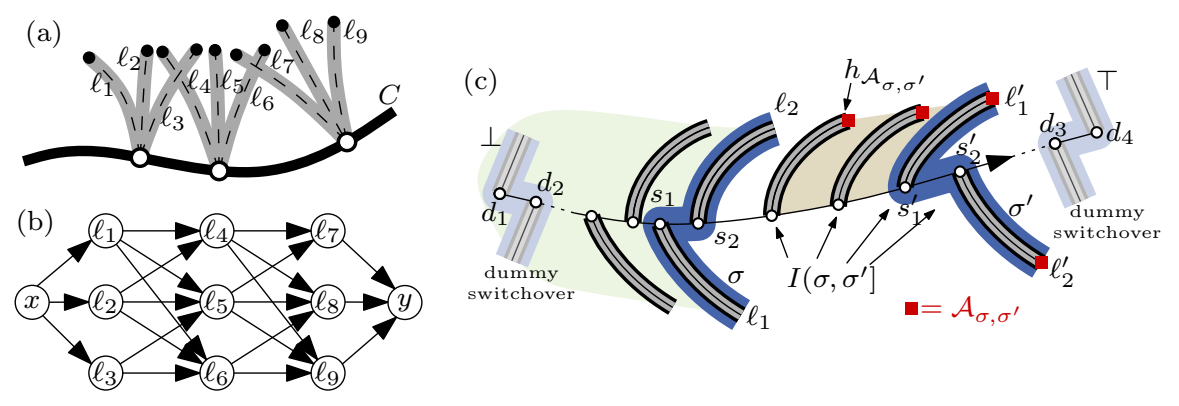

Fig. 7: Illustrations for labeling a single metro line. (a) A one-sided instance and (b) the acyclic directed graph $G$ based on its labels. (c) A two-sided instance with a labeling. The switchovers $\sigma^{\prime}$ and $\sigma$ separate the labeling into a two-sided and a one-sided instance.

If $I=(\{C\}, \mathcal{S}, \mathcal{K}, w)$ satisfies Assumption 13 we call MetroMapLabeling also SoftMetroLiNELABELING. We now introduce an algorithm that solves this problem in $O\left(n^{2} k^{4}\right)$ time, where $n=|\mathcal{S}|$ and $k=\max \left\{\left|\mathcal{K}_{s}\right| \mid s \in \mathcal{S}\right\}$. Note that $k$ is typically constant. We assume w.l.o.g. that $\mathcal{K}$ contains only candidates that do not intersect $C$.

Labels on One Side. We first assume that all candidate labels in $\mathcal{K}$ are assigned either to the left or to the right side of $C$; without loss of generality to the left side of $C$. For two stops $s, s^{\prime} \in \mathcal{S}$ we denote the instance restricted to the stops $\left\{s, s^{\prime}\right\} \cup\left\{s^{\prime \prime} \in \mathcal{S} \mid s<s^{\prime \prime}<s^{\prime}\right\}$ by $I\left[s, s^{\prime}\right]$. We denote the first stop of $C$ by $\underline{s}$ and the last stop by $\bar{s}$. The transitivity property directly yields the next lemma.

Lemma 1. Let $s, s^{\prime}$ and $s^{\prime \prime}$ be stops with $s<s^{\prime}<s^{\prime \prime}, \mathcal{L}$ be a labeling of $I\left[\underline{s}, s^{\prime}\right], \ell \in \mathcal{L} \cap \mathcal{K}_{s}$ and $\ell^{\prime} \in \mathcal{L} \cap \mathcal{K}_{s^{\prime}}$. Any $\ell^{\prime \prime} \in \mathcal{K}_{s^{\prime \prime}}$ intersecting $\ell$ also intersects $\ell^{\prime}$.

Proof. Recall for the proof that we assume that $I$ satisfies Assumptions 13.

Assume for the sake of contradiction that there is a candidate $\ell^{\prime \prime} \in \mathcal{K}_{s^{\prime \prime}}$ such that $\ell^{\prime \prime}$ intersects $\ell$ but not $\ell^{\prime}$; see Fig. 6(c). Since $\mathcal{L}$ is a labeling, the labels $\ell$ and $\ell^{\prime}$ do not intersect. Hence, neither $\ell$ and $\ell^{\prime}$ nor $\ell^{\prime}$ and $\ell^{\prime \prime}$ intersect. Since all three labels are assigned to the same side of $C$, the transitivity property holds, which directly contradicts that $\ell$ and $\ell^{\prime}$ do not intersect.

Hence, the lemma states that $\ell^{\prime}$ separates $\mathcal{L}$ from the candidates of the stops succeeding $s^{\prime}$. We use this observation as follows. Based on $\mathcal{K}$ we define a directed acyclic graph $G=(V, E)$; see Fig. 7(a)-(b). This graph contains a vertex $u$ for each candidate $\ell \in \mathcal{K}$ and the two vertices $x$ and $y$. We call $x$ the source and $y$ the target of $G$. Let $\ell_{u}$ denote the candidate that belongs to the vertex $u \in V \backslash\{x, y\}$. For each pair $u, v \in V \backslash\{x, y\}$ the graph contains the edge $(u, v)$ if and only if the stop of $\ell_{u}$ lies directly before the stop of $\ell_{v}$ and, furthermore, $\ell_{u}$ and $\ell_{v}$ do not intersect. Further, for each vertex $u$ of any candidate of $\underline{s}$ the graph contains the edge $(x, u)$, and for each vertex $u$ of any candidate of $\bar{s}$ the graph contains the edge $(u, y)$. For an edge $(u, v) \in E$ we define its cost $w_{e}$ as follows. For $u \neq x$ and $v \neq y$ we set $w_{e}=w_{1}\left(\ell_{v}\right)+w_{2}\left(\ell_{u}, \ell_{v}\right)$. For $x=u$ we set $w_{e}=w_{1}\left(\ell_{v}\right)$ and for $v=y$ we set $w_{e}=0$.

An $x$-y path $P \subseteq E$ in $G$ is a path in $G$ that starts at $x$ and ends at $y$. Its costs are $w(P)=\sum_{e \in P} w_{e}$. The $x$-y path with minimum costs among all $x$ - $y$ paths is the shortest $x-y$ path.

Lemma 2. For any $x-y$ path $P$ in $G$ there is a labeling $\mathcal{L}$ of $I$ with $w(P)=w(\mathcal{L})$ and for any labeling $\mathcal{L}$ of $I$ there is an $x-y$ path $P$ in $G$ with $w(P)=w(\mathcal{L})$.

Proof. Recall for the proof that we assume that $I$ satisfies Assumptions 13.

Let $P=\left(V_{P}, E_{P}\right)$ be an $x$-y path in $G$ and let $\mathcal{L}=\left\{\ell_{v} \in \mathcal{K} \mid v \in V_{P}\right\}$, where $V_{P}$ denotes the vertices of $P$ and $E_{P}$ the edges of $P$. We show that $\mathcal{L}$ is a labeling of $C$ with $w(\mathcal{L})=w(P)$. Obviously, for each stop $s \in \mathcal{S}$ the set $\mathcal{L}$ contains exactly one candidate $\ell \in \mathcal{K}_{s}$. By construction for each edge $(u, v) \in E_{P}$ the labels $\ell_{u}$ and $\ell_{v}$ do not intersect. Hence, by Lemma 1 the label $\ell_{v}$ cannot intersect any 
label $\ell \in \mathcal{L}$ of any stop that occurs before the stop of $\ell_{u}$. Hence, the set $\mathcal{L}$ is a labeling. Let $\ell_{1}, \ldots, \ell_{n}$ be the labels in $\mathcal{L}$ in the order of their stops. It holds

$$
w(P)=\sum_{e \in E_{P}} w_{e}=w_{1}\left(\ell_{1}\right)+\sum_{i=2}^{n}\left(w_{1}\left(\ell_{i}\right)+w_{2}\left(\ell_{i-1}, \ell_{i}\right)\right)=w(\mathcal{L})
$$

Now, let $\mathcal{L}$ be an arbitrary labeling of $C$. We show that there is an $x-y$ path $P$ with $w(P)=w(\mathcal{L})$. Let $s$ and $s^{\prime}$ be two consecutive stops with $s<s^{\prime}$ and let $\ell$ and $\ell^{\prime}$ be the corresponding labels in $\mathcal{L}$. Since $\ell$ and $\ell^{\prime}$ do not intersect, the corresponding vertices $u$ and $u^{\prime}$ of $\ell$ and $\ell^{\prime}$ are adjacent in $G$. Hence, the labels in $\mathcal{L}$ induce a path $P$ in $G$. Let $\ell_{1}, \ldots, \ell_{n}$ be the labels in $\mathcal{L}$ in the order of their stops. Using Equation 1 we obtain $w(P)=w(\mathcal{L})$.

The lemma in particular proves that a shortest $x-y$ path $P$ in $G$ corresponds with an optimal labeling of $I$. Due to [4. Chapter 24], $P$ can be constructed in $O(|V|+|E|)$ time using a dynamic programming approach, which we call MinPath. In particular MinPath considers each edge only once. There are $O(n \cdot k)$ vertices in $G$ and each vertex has at most $k$ incoming edges, which implies that there are $O\left(n \cdot k^{2}\right)$ edges. Since MinPath considers each edge only once, we compute the edges of $G$ on demand, which saves storage.

Theorem 2. If I is one-sided, SoftMetroLineLABELING can be optimally solved in $O\left(n k^{2}\right)$ time and $O(n k)$ space.

Labels on Both Sides. If candidates lie on both sides of the metro line, we solve the problem utilizing the algorithm for the one-sided case.

Consider a labeling $\mathcal{L}$ of $I$ and let $\sigma, \sigma^{\prime}$ be two switchovers in $\mathcal{L}$ such that $\sigma$ lies before $\sigma^{\prime}$ and no other switchover lies in between both; see Fig. 7 (c). Roughly spoken, $\sigma$ and $\sigma^{\prime}$ induce a two-sided instance that lies before $\sigma$ and a one-sided instance that lies in between both switchovers $\sigma$ and $\sigma^{\prime}$.

Lemma 3. Let $s, s_{1}^{\prime}, s_{2}^{\prime}$ and $s^{\prime \prime}$ be stops with $s<s_{1}^{\prime}<s_{2}^{\prime}<s^{\prime \prime} ; s_{1}^{\prime}$ and $s_{2}^{\prime}$ are consecutive. Let $\mathcal{L}$ be a labeling of $I\left[\underline{s}, s_{2}^{\prime}\right], \ell \in \mathcal{L} \cap \mathcal{K}_{s}, \ell_{1}^{\prime} \in \mathcal{L} \cap \mathcal{K}_{s_{1}^{\prime}}, \ell_{2}^{\prime} \in \mathcal{L} \cap \mathcal{K}_{s_{2}^{\prime}}$ s.t. $\left(\ell_{1}^{\prime}, \ell_{2}^{\prime}\right)$ is a switchover. Any $\ell^{\prime \prime} \in \mathcal{K}_{s^{\prime \prime}}$ intersecting $\ell$ intersects $\ell_{1}^{\prime}$ or $\ell_{2}^{\prime}$.

Proof. Recall for the proof that we assume that $I$ satisfies Assumptions 113 .

Assume for the sake of contradiction that there is a label $\ell^{\prime \prime} \in \mathcal{K}_{s^{\prime \prime}}$ such that $\ell^{\prime \prime}$ intersects $\ell$ without intersecting $\ell_{1}^{\prime}$ and $\ell_{2}^{\prime}$. Since $\ell$ and $\ell^{\prime \prime}$ intersect each other, due to Assumption 1 both are assigned to the same side of $C$; w.l.o.g., let $\ell$ and $\ell^{\prime \prime}$ be assigned to the left hand side of $C$. Further, w.l.o.g., let $\ell_{1}^{\prime}$ be a left candidate and $\ell_{2}^{\prime}$ a right candidate; analogous arguments hold for the opposite case. Since $\mathcal{L}$ is a labeling, the labels $\ell$ and $\ell_{1}^{\prime}$ do not intersect. Hence, neither $\ell$ and $\ell_{1}^{\prime}$ nor $\ell_{1}^{\prime}$ and $\ell^{\prime \prime}$ intersect. Since $\ell, \ell_{1}^{\prime}$ and $\ell^{\prime \prime}$ are assigned to the same side of $C$, the transitivity property must hold. However, this contradicts that $\ell$ and $\ell^{\prime \prime}$ intersect.

Hence, the lemma yields that for the one-sided instance we can choose any labeling; as long as this labeling does not intersect any label of $\sigma$ or $\sigma^{\prime}$, it composes with $\sigma, \sigma^{\prime}$ and the labeling of the two-sided instance to one labeling for the instance up to $\sigma^{\prime}$. We use that observation as follows.

Let $\sigma=\left(\ell_{1}, \ell_{2}\right)$ and $\sigma^{\prime}=\left(\ell_{1}^{\prime}, \ell_{2}^{\prime}\right)$ be two switchovers in $\mathcal{W}$. Let $s_{1}$ and $s_{2}$ be the stops of $\ell_{1}$ and $\ell_{2}$, and let $s_{1}^{\prime}$ and $s_{2}^{\prime}$ be the stops of $\ell_{1}^{\prime}$ and $\ell_{2}^{\prime}$, respectively; see Fig. 7 (c). We assume that $\sigma<\sigma^{\prime}$, i.e., $s_{1}<s_{1}^{\prime}$. Let $I\left(\sigma, \sigma^{\prime}\right]$ be the instance restricted to the stops $\left\{s \in \mathcal{S} \mid s_{2}<s<s_{1}^{\prime}\right\} \cup\left\{s_{1}^{\prime}, s_{2}^{\prime}\right\}$, where $\left(\sigma, \sigma^{\prime}\right]$ indicates that the stops of $\sigma^{\prime}$ belong to that instance, while the stops of $\sigma$ do not.

The switchovers $\sigma$ and $\sigma^{\prime}$ are compatible if $\ell_{2}$ and $\ell_{1}^{\prime}$ are assigned to the same side of $C$, and there is a labeling for $I\left[s_{1}, s_{2}^{\prime}\right]$ such that it contains $\ell_{1}, \ell_{2}, \ell_{1}^{\prime}$ and $\ell_{2}^{\prime}$ and, furthermore, $\sigma$ and $\sigma^{\prime}$ are the only switchovers in that labeling. Let $\mathcal{L}$ be the optimal labeling among those labelings. We denote the labeling $\mathcal{L} \backslash\left\{\ell_{1}, \ell_{2}\right\}$ of $I\left(\sigma, \sigma^{\prime}\right]$ by $\mathcal{A}_{\sigma, \sigma^{\prime}}$. Utilizing Theorem 2 , we obtain $\mathcal{A}_{\sigma, \sigma^{\prime}}$ in $O\left(n \cdot k^{2}\right)$ time.

For any labeling $\mathcal{L}$ of an instance $J$ let $h_{\mathcal{L}} \in \mathcal{L}$ be the label of the first stop in $J$ and let $t_{\mathcal{L}} \in \mathcal{L}$ be the label of the last stop in $J ; h_{\mathcal{L}}$ is the head and $t_{\mathcal{L}}$ is the tail of $\mathcal{L}$. For technical reasons we extend $\mathcal{S}$ 
by the dummy stops $d_{1}, d_{2}, d_{3}$ and $d_{4}$ such that $d_{1}<d_{2}<s<d_{3}<d_{4}$ for any stop $s \in \mathcal{S}$. For $d_{1}$ and $d_{2}$ we introduce the dummy switchover $\perp$ and for $d_{3}$ and $d_{4}$ the dummy switchover $T$. We define that $\perp$ and $T$ are compatible to all switchovers in $\mathcal{W}$ and that $\perp$ and $T$ are compatible, if there is a one-sided labeling for $I$. Conceptually, each dummy switchover consists of two labels that are assigned to both sides of $C$. Further, neither $\perp$ nor $T$ has any influence on the cost of a labeling. Hence, w.l.o.g. we assume that they are contained in any labeling.

Similar to the one-sided case we define a directed acyclic graph $G^{\prime}=\left(V^{\prime}, E^{\prime}\right)$. This graph contains a vertex $u$ for each switchover $\mathcal{W} \cup\{\perp, \top\}$. Let $\sigma_{u}$ denote the switchover that belongs to the vertex $u \in V$. In particular let $x$ denote the vertex of $\perp$ and $y$ denote the vertex of $\top$. For each pair $u, v \in V$ the graph contains the edge $(u, v)$ if and only if $\sigma_{u}$ and $\sigma_{v}$ are compatible and $\sigma_{u}<\sigma_{v}$. The cost $w_{e}$ of an edge $e=(u, v)$ in $G^{\prime}$ is $w_{e}=w\left(\mathcal{A}_{\sigma_{u}, \sigma_{v}}\right)+w_{3}\left(\sigma_{u}, \sigma_{v}\right)+w_{2}\left(\ell_{2}^{u}, h_{\mathcal{A}_{\sigma_{u}, \sigma_{v}}}\right)$, where $\sigma_{u}=\left(\ell_{1}^{u}, \ell_{2}^{u}\right)$. In the special case that $\sigma_{u}$ and $\sigma_{v}$ share a stop, we set $w_{e}=w_{3}\left(\sigma_{u}, \sigma_{v}\right)+w_{2}\left(\ell_{1}^{v}, \ell_{2}^{v}\right)+w_{1}\left(\ell_{2}^{v}\right)$, where $\sigma_{v}=\left(\ell_{1}^{v}, \ell_{2}^{v}\right)$.

Let $P$ be an $x-y$ path in $G^{\prime}$ and let $e_{1}=\left(x=v_{0}, v_{1}\right), e_{2}=\left(v_{1}, v_{2}\right), \ldots, e_{l}=\left(v_{l-1}, v_{l}=y\right)$ be the edges of $P$. For a vertex $v_{i}$ of $P$ with $0 \leq i \leq l$ we write $\sigma_{i}$ instead of $\sigma_{v_{i}}$. We denote the set $\bigcup_{i=1}^{l} A_{\sigma_{i-1}, \sigma_{i}}$ by $\mathcal{L}_{P}$.

Lemma 4. a) The graph $G^{\prime}$ has an $x-y$ path if and only if I has a labeling. b) Let $P$ be a shortest $x-y$ path in $G^{\prime}$, then $\mathcal{L}_{P}$ is an optimal labeling of $I$.

Proof. Recall for the proof that we assume that $I$ satisfies Assumption 13.

By construction of $G^{\prime}$, it directly follows that $G^{\prime}$ has an $x-y$ path $P$ if and only if $I$ has a labeling $\mathcal{L}$. We first show that $\mathcal{L}_{P}$ is a labeling of $I$ with $w\left(\mathcal{L}_{P}\right)=w(P)$. Afterwards we prove that for any labeling $\mathcal{L}$ of $I$ it holds $w(\mathcal{L}) \geq w\left(\mathcal{L}_{P}\right)$.

Let $e_{1}=\left(v_{0}, v_{1}\right), e_{2}=\left(v_{1}, v_{2}\right), \ldots, e_{l}=\left(v_{l-1}, v_{l}\right)$ be the edges of $P$ with $v_{0}=x$ and $v_{l}=y$. For a vertex $v_{i}$ with $0 \leq i \leq l$ we write $\sigma_{i}$ instead of $\sigma_{v_{i}}$. We show by induction over $m \in \mathbb{N}$ with $1 \leq m \leq l$ that

$$
\mathcal{L}_{m}:=\bigcup_{i=1}^{m} \mathcal{A}_{\sigma_{i-1}, \sigma_{i}}
$$

is a labeling of $I\left(\sigma_{0}, \sigma_{m}\right]$ with $w\left(\mathcal{L}_{m}\right)=\sum_{i=1}^{m} w_{e_{i}}$. Altogether this implies that $\mathcal{L}_{P}=\mathcal{L}_{l}$ is a labeling with $w\left(\mathcal{L}_{P}\right)=w(P)$.

For $m=1$ we have $\mathcal{L}_{1}=\mathcal{A}_{\sigma_{0}, \sigma_{1}}$. By the construction of $G^{\prime}$ the set $\mathcal{A}_{\sigma_{0}, \sigma_{1}}$ is a labeling. Since $\sigma_{0}=\left(\ell_{0}, \ell_{1}\right)$ is a dummy switchover we have $w_{3}\left(\sigma_{0}, \sigma_{1}\right)=0$ and $w_{2}\left(\ell_{2}, h_{\mathcal{A}_{\sigma_{0}, \sigma_{1}}}\right)=0$. Hence, it holds $w\left(\mathcal{A}_{\sigma_{0}, \sigma_{1}}\right)=w_{e_{1}}$.

Now, consider the set $\mathcal{L}_{m}$ for $m>1$. We first argue that $\mathcal{L}_{m}$ is a labeling of $I\left(\sigma_{0}, \sigma_{m}\right]$. By induction the set $\mathcal{L}_{m-1} \subseteq \mathcal{L}_{m}$ is a labeling of $I\left(\sigma_{0}, \sigma_{m-1}\right]$. Further, by construction the set $\mathcal{L}_{m} \backslash \mathcal{L}_{m-1}=A_{\sigma_{m-1}, \sigma_{m}}$ is a labeling of the instance $I\left(\sigma_{m-1}, \sigma_{m}\right]$. Since $\sigma_{m-1}$ and $\sigma_{m}$ are compatible, no label of $\mathcal{L}_{m} \backslash \mathcal{L}_{m-1}$ intersects any label of $\sigma_{m-1}$. Then by Lemma 3 no two labels in $\mathcal{L}_{m}$ intersect each other.

We now show that $w\left(\mathcal{L}_{m}\right)=\sum_{i=1}^{m} w_{e_{i}}$. By induction we have $w\left(\mathcal{L}_{m-1}\right)=\sum_{i=1}^{m-1} w_{e_{i}}$. Since $\mathcal{L}_{m}=$ $\mathcal{L}_{m-1} \cup \mathcal{A}_{\sigma_{m-1}, \sigma_{m}}$ it holds

$$
w\left(\mathcal{L}_{m}\right)=w\left(\mathcal{L}_{m-1} \cup \mathcal{A}_{\sigma_{m-1}, \sigma_{m}}\right) .
$$

We distinguish two cases. First assume that $\sigma_{m-1}$ and $\sigma_{m}$ do not have any stop in common. Let $\sigma_{m-1}=$ $\left(\ell_{1}^{m-1}, \ell_{2}^{m-1}\right)$, then we derive from Equation 2 )

$$
\begin{aligned}
w\left(\mathcal{L}_{m}\right) & =w\left(\mathcal{L}_{m-1} \cup \mathcal{A}_{\sigma_{m-1}, \sigma_{m}}\right) \\
& =w\left(\mathcal{L}_{m-1}\right)+w\left(\mathcal{A}_{\sigma_{m-1}, \sigma_{m}}\right)+w_{3}\left(\sigma_{m-1}, \sigma_{m}\right)+w_{2}\left(\ell_{2}^{m-1}, h_{\mathcal{A}_{\sigma_{m-1}, \sigma_{m}}}\right) \\
& \stackrel{(I)}{=} w\left(\mathcal{L}_{m-1}\right)+w_{e_{m}} \stackrel{(I I)}{=} \sum_{i=1}^{m} w_{e_{i}} .
\end{aligned}
$$

Equality (I) holds due to the definition of $w_{e_{m}}$ and Equality (II) is by induction true. Now assume that $\sigma_{m-1}$ and $\sigma_{m}$ have a stop in common. Let $\sigma_{m}=\left(\ell_{1}^{m}, \ell_{2}^{m}\right)$, then we derive from Equation (2) 


$$
\begin{aligned}
& w\left(\mathcal{L}_{m}\right)=w\left(\mathcal{L}_{m-1} \cup \mathcal{A}_{\sigma_{m-1}, \sigma_{m}}\right) \\
& \quad=w\left(\mathcal{L}_{m-1}\right)+w_{3}\left(\sigma_{m-1}, \sigma_{m}\right)+w_{2}\left(\ell_{1}^{m}, \ell_{2}^{m}\right)+w_{1}\left(\ell_{2}^{m}\right) \\
& \quad \stackrel{(I I I)}{=} w\left(\mathcal{L}_{m-1}\right)+w_{e_{m}} \stackrel{(I V)}{=} \sum_{i=1}^{m} w_{e_{i}} .
\end{aligned}
$$

Equality (III) holds due to the definition of $w_{e_{m}}$ and Equality (IV) is by induction true. Altogether we obtain that $\mathcal{L}_{l}$ is a labeling with $w\left(\mathcal{L}_{l}\right)=w(P)$.

Finally we show, that there is no other labeling $\mathcal{L}$ with $w(\mathcal{L})<w\left(\mathcal{L}_{P}\right)$. Assume for the sake of contradiction that there is such a labeling $\mathcal{L}$. Let $\perp=\sigma_{0}, \sigma_{1}, \ldots, \sigma_{l}=\top$ be the switchovers in $\mathcal{L}$, such that $\sigma_{i}<\sigma_{i+1}$ for each $0 \leq i<l$. We observe that two consecutive switchovers $\sigma_{i}$ and $\sigma_{i+1}$ are compatible. Hence, for any $i$ with $1 \leq i \leq l$ there is an edge $e_{i}=(u, v)$ in $G^{\prime}$ with $\sigma_{u}=\sigma_{i-1}$ and $\sigma_{v}=\sigma_{i+1}$. Consequently, the edges $e_{1}, \ldots, e_{l}$ form an $x$-y path $Q$. By the first two claims of this lemma, there is a labeling $\mathcal{L}_{Q}$ of $I$ with $w(Q)=w\left(\mathcal{L}_{Q}\right)$. Since $P$ is a shortest $x$-y path it holds

$$
w(\mathcal{L})<w\left(\mathcal{L}_{P}\right)=w(P) \leq w(Q)=w\left(\mathcal{L}_{Q}\right)
$$

We now show that $w\left(\mathcal{L}_{Q}\right) \leq w(\mathcal{L})$ deriving a contradiction. To that end recall that

$$
\mathcal{L}_{Q}=\bigcup_{i=1}^{l} \mathcal{A}_{\sigma_{i-1}, \sigma_{i}} .
$$

For $1 \leq i \leq l$ the set $\mathcal{A}_{\sigma_{i-1}, \sigma_{i}}$ is an optimal labeling of the instance $I\left(\sigma_{i-1}, \sigma_{i}\right]$ such that $\mathcal{A}_{\sigma_{i-1}, \sigma_{i}} \cup \sigma_{i-1}$ is a labeling and $\sigma_{i}$ is the only switchover contained in $\mathcal{A}_{\sigma_{i-1}, \sigma_{i}}$. Let $\mathcal{L}_{i} \subseteq \mathcal{L}$ and $\mathcal{L}_{i}^{Q} \subseteq \mathcal{L}_{Q}$ be the labelings restricted to $I\left(\sigma_{i-1}, \sigma_{i}\right]$. In particular we have $\mathcal{L}_{i}^{Q}=\mathcal{A}_{\sigma_{i-1}, \sigma_{i}}$ and $\sigma_{i}$ is the only switchover in $\mathcal{L}_{i}$. If we had $w\left(\mathcal{L}_{Q}\right)>w(\mathcal{L})$, there must be two consecutive switchovers $\sigma_{i-1}$ and $\sigma_{i}$ such that $w\left(\mathcal{L}_{i}\right)<w\left(\mathcal{L}_{i}^{Q}\right)$. However, this contradicts the optimality of $\mathcal{A}_{\sigma_{i-1}, \sigma_{i}}$. Consequently, it holds $w\left(\mathcal{L}_{Q}\right) \leq w(\mathcal{L})$ yielding the claimed contradiction.

By Lemma 4 a shortest $x$-y path $P$ in $G^{\prime}$ corresponds with an optimal labeling $\mathcal{L}$ of $C$, if this exists. Using MinPath we construct $P$ in $O\left(\left|V^{\prime}\right|+\left|E^{\prime}\right|\right)$ time. Since $\mathcal{W}$ contains $O\left(n k^{2}\right)$ switchovers, the graph $G^{\prime}$ contains $O\left(n k^{2}\right)$ vertices and $O\left(n^{2} k^{4}\right)$ edges. As MinPath considers each edge only once, we compute the edges of $G^{\prime}$ on demand, which needs $O\left(n k^{2}\right)$ storage. We compute the costs of the incoming edges of a vertex $v \in V^{\prime}$ utilizing the one-sided case. Proceeding naively, we need $O\left(n k^{2}\right)$ time per edge, which yields $O\left(n^{3} k^{6}\right)$ time in total.

Reusing already computed information, we improve that result as follows. Let $\left(u_{1}, v\right), \ldots,\left(u_{k}, v\right)$ denote the incoming edges of $v$ such that $\sigma_{u_{1}} \leq \cdots \leq \sigma_{u_{k}}$, i.e., the stop of $\sigma_{u_{i}}$ 's first label does not lie after the stop of $\sigma_{u_{j}}$ 's first label with $i<j$. Further, let $\sigma_{v}=\left(\ell_{v}^{1}, \ell_{v}^{2}\right)$ and let $G_{i}$ be the graph for the one-sided instance $I\left[s_{i}, s\right]$ considering only candidates that lie on the same side as $\ell_{v}^{1}$, where $s_{i}$ is the stop of the second label of $\sigma_{u_{i}}$ and $s$ is the stop of $\ell_{v}^{1}$. Let $P_{i}$ be the shortest $x_{i}$ - $y_{i}$ path in $G_{i}$, where $x_{i}$ and $y_{i}$ denote the source and target of $G_{i}$, respectively. We observe that excluding the source and target, the graph $G_{i}$ is a sub-graph of $G_{1}$ for all $1 \leq i \leq k$. Further, since a sub-path of a shortest path is also a shortest path among all paths having the same end vertices, we can assume without loss of optimality that when excluding $x_{i}$ and $y_{i}$ from $P_{i}$, the path $P_{i}$ is a sub path of $P_{1}$ for all $1 \leq i \leq k$. We therefore only need to compute $G_{1}$ and $P_{1}$ and can use sub-paths of $P_{1}$ in order to gain the costs of all incoming edges of $v$. Hence, we basically apply for each vertex $v \in V$ the algorithm for the one-sided case once using $O\left(n k^{2}\right)$ time per vertex. We then can compute the costs in $O(1)$ time per edge, which yields the next result.

Theorem 3. SoftMetroLineLabeling can be optimally solved in $O\left(n^{2} k^{4}\right)$ time and $O\left(n k^{2}\right)$ space. 


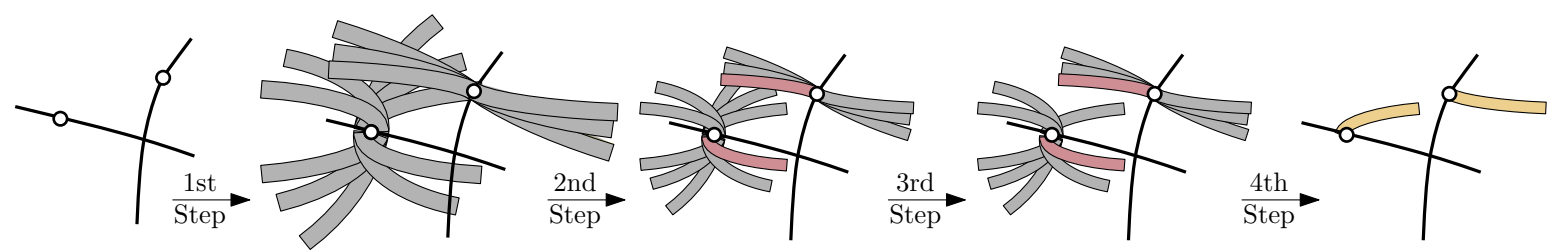

Fig. 8: Schematic illustration of the presented workflow. 1st Step: generation of candidates. 2nd Step: scaling and creation of initial labeling (red labels). 3rd Step: pre-selection of candidates. 4th Step: Solving the metro lines independently.

\section{Cost Function}

In this section we motivate the cost function introduced in Section 4 For a given metro map $(\{C\}, \mathcal{S})$ that consists of a single metro line $C$, and generated candidates $\mathcal{K}$, we rate each labeling $\mathcal{L} \subseteq \mathcal{K}$ using the following cost function:

$$
w(\mathcal{L})=\sum_{\ell \in \mathcal{L}} w_{1}(\ell)+\sum_{\left(\ell_{1}, \ell_{2}\right) \in P_{\mathcal{L}}} w_{2}\left(\ell_{1}, \ell_{2}\right)+\sum_{\left(\sigma_{1}, \sigma_{2}\right) \in \Gamma_{\mathcal{L}}} w_{3}\left(\sigma_{1}, \sigma_{2}\right),
$$

where $w_{1}: \mathcal{L} \rightarrow \mathbb{R}$ rates a single label, $w_{2}: P_{\mathcal{L}} \rightarrow \mathbb{R}$ rates two consecutive labels and $w_{3}: \Gamma_{\mathcal{L}} \rightarrow \mathbb{R}$ rates two consecutive switchovers; see Assumption 3 The definition of this function relies on the following considerations, which are based on Imhof's "general principles and requirements" for map labeling [8].

To respect that some (e.g., steep and highly curved) labels are more difficult to read than others, we introduce a cost $w_{1}(\ell)$ for each candidate label $\ell$.

Imhof further notes that "names should assist directly in revealing spatial situation" and exemplifies this principle with maps that show text only while still conveying the most relevant geographic information. To transfer this idea to metro maps, we favor solutions where the labels for each two consecutive stops on a metro line have similar properties. That is, the two labels should be placed on the same side of the line and their slopes and curvatures should be similar. In a map satisfying this criterion, a user need not find the point-text correspondence on a one-to-one basis. Instead, the user can identify metro lines and sequences of stops based on label groups, which, for example, makes it easier to count the stops till a destination. (Of course, this is also an improvement in terms of legibility.) In our model, we consider the similarity of consecutive labels by introducing a cost $w_{2}\left(\ell_{1}, \ell_{2}\right)$ for each pair $\left(\ell_{1}, \ell_{2}\right)$ of candidates that belong to consecutive stops on $C$. We penalize consecutive candidates that lie on opposite sides of the metro line, because those disturb the overall label placement. We add this cost to the objective value of a solution if both candidates are selected. Since we minimize the total costs of the solution, the cost for a pair of candidates should be low if they are similar. Further, if $C$ has labels that do not lie on the same side of $C$, the implied switchovers should occur in regular distances and not cluttered. Hence, for each pair $\sigma_{1}$ and $\sigma_{2}$ of two consecutive switchovers in a solution, we add a cost $w_{3}\left(\sigma_{1}, \sigma_{2}\right)$ to the objective value of the solution that depends on the distance between $\sigma_{1}$ and $\sigma_{2}$; the smaller the distance, the greater the cost of $w_{3}\left(\sigma_{1}, \sigma_{2}\right)$.

We now describe more precisely how we defined $w_{1}, w_{2}$ and $w_{3}$ for our evaluation. The definitions depend on the applied labeling style.

Curved Labels. Using CuRvedStyle for the labels we define the cost functions as follows. For a label $\ell \in \mathcal{K}$ let $p_{\ell}^{1}=\left(x_{\ell}^{1}, y_{\ell}^{1}\right)$ be the start point of $\ell$ and let $p_{\ell}^{2}=\left(x_{\ell}^{2}, y_{\ell}^{2}\right)$ be the end point of $\ell$; recall that we derived the labels from Bézier curves. Let $\boldsymbol{v}_{\ell}$ be the vector connecting $p_{\ell}^{1}$ with $p_{\ell}^{2}$ and let $\alpha \in[0,2 \pi]$ denote the angle of $\boldsymbol{v}_{\ell}$. We define

$$
\delta_{\ell}= \begin{cases}\alpha_{\ell} & 0 \leq \alpha \leq \frac{\pi}{2} \\ \pi-\alpha_{\ell} & \frac{\pi}{2}<\alpha \leq \pi \\ \alpha_{\ell}-\pi & \pi<\alpha \leq \frac{3}{2} \pi \\ 2 \pi-\alpha_{\ell} & \frac{3}{2} \pi<\alpha<2 \pi\end{cases}
$$


Hence, the angle $\delta_{\ell}$ is a measure for how horizontal the vector $\boldsymbol{v}_{\ell}$ is, whereby the smaller the value of $\delta_{\ell}$, the more horizontal is $\boldsymbol{v}_{\ell}$.

We defined the cost function $w_{1}$ rating a single label $\ell \in \mathcal{K}$ to be $w_{1}=10 \cdot \delta_{\ell}$. Hence, we penalize steep labels. We defined $w_{2}$ rating two consecutive labels $\ell_{1}$ and $\ell_{2}$ as follows. If $\ell_{1}$ and $\ell_{2}$ point into different $x$-direction, $w_{2}$ is 150 . Else, if $\ell_{1}$ and $\ell_{2}$ are switchovers, the cost $w_{2}$ is 0 . In all other cases, $w_{2}$ is the difference between the angle $\alpha_{\ell_{1}}$ and $\alpha_{\ell_{2}}$. Hence, in the latter case we penalize labels that are differently aligned. Finally, $w_{3}$ rating two switchovers $\sigma_{1}$ and $\sigma_{2}$ of the same line is defined as

$w_{3}=\frac{200}{d_{\sigma_{1}, \sigma_{2}}}$, where $d_{\sigma_{1}, \sigma_{2}}$ is the number of stops in between $\sigma_{1}$ and $\sigma_{2}$. In particular $w_{3}$ effects that a labeling with equally sized sequences of labels lying on the same side of their metro line are rated better than a labeling where the sequences are sized irregularly.

Octilinear Labels. Using OCTILINStyle for the labels we define the cost functions as follows. Recall that we use OctilinStyle for octilinear metro maps. For a label $\ell$ let $l_{\ell}$ be the segment on which its stop is placed. If $l_{\ell}$ is horizontal, but $\ell$ is not diagonal, we set $w_{1}=200$. If $l_{\ell}$ is vertical or diagonal, but $\ell$ is not horizontal, we set $w_{1}=100$. In all other cases we set $w_{1}=0$. The functions $w_{2}$ and $w_{3}$ are defined in the same way as for CurvedStyle.

\section{Multiple Metro Lines}

In this section we consider the problem that we are given a metro map $(\mathcal{M}, \mathcal{S})$ consisting of multiple metro lines. We present an algorithm that creates a labeling for $(\mathcal{M}, \mathcal{S})$ in two phases. Each phase is divided into two steps; see Fig. 8 for a schematic illustration. In the first phase the algorithm creates the set $\mathcal{K}$ of label candidates and ensures that there exists at least one labeling for the metro map. In the second phase it then computes a labeling $\mathcal{L}$ for $(\mathcal{M}, \mathcal{S}, \mathcal{K})$. To that end it makes use of the labeling algorithm for a single metro line; see Section 4 In order to rate $\mathcal{L}$, we extend the cost function $w$ for a single metro line on multiple metro lines, i.e., for any $\mathcal{L} \subseteq \mathcal{K}$ we require

$$
w(\mathcal{L})=\sum_{C \in \mathcal{M}} w(C) \text { with } w(C)=\sum_{\ell \in \mathcal{L}_{C}} w_{1}(\ell)+\sum_{\left(\ell_{1}, \ell_{2}\right) \in P_{\mathcal{L}_{C}}} w_{2}\left(\ell_{1}, \ell_{2}\right)+\sum_{\left(\sigma_{1}, \sigma_{2}\right) \in \Gamma_{\mathcal{L}_{C}}} w_{3}\left(\sigma_{1}, \sigma_{2}\right),
$$

where $\mathcal{L}_{C}=\mathcal{L} \cap \mathcal{K}_{C}$ is the labeling restricted to metro line $C \in \mathcal{M}, w_{1}: \mathcal{L}_{C} \rightarrow \mathbb{R}$ rates a single label, $w_{2}: P_{\mathcal{L}_{C}} \rightarrow \mathbb{R}$ rates two consecutive labels and $w_{3}: \Gamma_{\mathcal{L}_{C}} \rightarrow \mathbb{R}$ rates two consecutive switchovers of $C$. In particular $w$ satisfies Assumption 3 for a single metro line.

Altogether, the workflow yields a heuristic that relies on the conjecture that using optimal algorithms in single steps is sufficient to obtain good labelings. In our evaluation we call that approach DPALG.

\subsection{First Phase - Candidate Generation}

First, we create the label candidates $\mathcal{K}$. We then enforce that there is a labeling $\mathcal{L}$ for the given instance $I=(\mathcal{M}, \mathcal{S}, \mathcal{K})$.

1st Step - Candidate Creation. Depending on the labeling style, we generate a discrete set of candidate labels for every stop. Hence, we are now given the instance $(\mathcal{M}, \mathcal{S}, \mathcal{K}, w)$. In particular we assume that each candidate $\ell \in \mathcal{K}$ is assigned to one side of its metro line $C$, namely to the left or right side of $C$, and, furthermore, $w$ is a cost function satisfying Assumption 3 for each metro line $C$.

2nd Step - Scaling. Since each stop of a metro map must be labeled, we first apply a transformation on the given candidates to ensure that there is at least one labeling of the metro map. To that end we first determine for each stop $s \in \mathcal{S}$ of each metro line $C \in \mathcal{M}$ two candidates of $\mathcal{K}_{s}$ that are assigned to opposite sides of $C$. More specifically, among all candidates in $\mathcal{K}_{s}$ that are assigned to the right hand side of $C$ and that do not intersect any metro line of $\mathcal{M}$, we take that candidate $\ell_{\mathrm{R}} \in \mathcal{K}_{s}$ with 
minimum costs, i.e., $w_{1}(\ell)$ is minimal. If such a candidate does not exist, because each label of $\mathcal{K}_{s}$ intersects at least one metro line, we take a pre-defined label $\ell_{\mathrm{R}} \in \mathcal{K}_{s}$ that is assigned to the right hand side of $C$. In the same manner we choose a candidate $\ell_{\mathrm{L}} \in \mathcal{K}_{s}$ that is assigned to the left hand side of $C$. Let $D_{s}=\left\{\ell_{\mathrm{R}}, \ell_{\mathrm{L}}\right\}$, we now enforce that there is a labeling $\mathcal{L}$ of $I$ such that for each stop $s \in \mathcal{S}$ it contains a label of $D_{s}$.

We check whether the set $\bigcup_{s \in \mathcal{S}} D_{s}$ admits a labeling $\mathcal{L}$ for $I$. Later, we describe more specifically how to do this. If $\mathcal{L}$ exists, we continue with the third step of the algorithm using $I$ and $\mathcal{L}$ as input. Otherwise, we scale all candidates of $\mathcal{K}$ smaller by a constant factor and repeat the described procedure. Sampling a pre-defined scaling range $\left[x_{\min }, x_{\max }\right]$, we find in that manner a scaling factor $x \in\left[x_{\min }, x_{\max }\right]$ for the candidates that admits a labeling $\mathcal{L}$ of $I$. We choose $x$ as large as possible. If we could not find $x$, e.g, because we have chosen $\left[x_{\min }, x_{\max }\right]$ or the sampling not appropriately, we abort the algorithm, stating that the algorithm could not find a labeling.

Next, we describe how to check whether there is a labeling for $\bigcup_{s \in \mathcal{S}} D_{s}$. Since for each $s \in \mathcal{S}$ the set $D_{s}$ contains two candidates, we can make use of a 2SAT formulation to model the labeling problem. For each stop $s \in \mathcal{S}$ and each candidate $\ell \in D_{s}$, we introduce the Boolean variable $x_{\ell}$. Those Boolean variables induce the set $\mathcal{L}=\left\{\ell \in \bigcup_{s \in S} D_{s} \mid x_{\ell}\right.$ is true $\}$. The following formulas are satisfiable if and only if $\mathcal{L}$ is a labeling of $\mathcal{M}$.

$$
\begin{aligned}
\neg x_{\ell} & \forall s \in \mathcal{S} \forall \ell \in D_{s} \text { s.t. } \exists C \in \mathcal{M} \text { that intersects } \ell \\
\neg x_{\ell} \vee \neg x_{\ell^{\prime}} & \forall \ell, \ell^{\prime} \in \bigcup_{s \in S} D_{s} \text { s.t. } \ell \text { and } \ell^{\prime} \text { intersect. } \\
x_{\ell} \vee x_{\ell^{\prime}}, \neg x_{\ell} \vee \neg x_{\ell^{\prime}} & \forall s \in \mathcal{S} \forall \ell, \ell^{\prime} \in D_{s}
\end{aligned}
$$

The first formula ensures that there is no label of the solution that intersects any metro line. The second one avoids overlaps between labels, while the two last formulas enforce that for each stop $s \in \mathcal{S}$ there is exactly one label of $D_{s}$ that is contained in the solution.

According to 2 in linear time with respect to the number of variables and formulas, the satisfiability can be checked. We introduce $O(n)$ variables and instantiate the second formula $O\left(n^{2}\right)$ times, because each pair of candidates may overlap. The remaining formulas are instantiated in $O\left(n^{2}\right)$ time. Hence, the total running time is in $O\left(n^{2} \cdot t\right)$, where $t$ denotes the number of scaling steps.

\subsection{Second Phase - Candidate Selection}

We assume that we are given the scaled instance $I=(\mathcal{M}, \mathcal{S}, \mathcal{K})$ and the labeling $\mathcal{L}$ for $I$ of the previous phase. We apply a pre-selection on the candidates by discarding candidates such that no two stops' candidates of different metro lines intersect and each metro line satisfies Assumption 1 and Assumption 2. We never remove a label in $\mathcal{L}$ from the candidate set, however, to ensure that there is always a feasible solution. Finally, considering the metro lines independently, we select for each stop a candidate to be its label using the dynamic program described in Section 4 .

3th Step - Candidate Pre-Selection. We first ensure that I satisfies Assumption 1 and Assumption 2. If two candidates $\ell$ and $\ell^{\prime}$ of the same metro line $C$ intersect and if they are assigned to opposite sides of $C$, we delete one of both labels as follows. If $\ell \in \mathcal{L}$, we delete $\ell^{\prime}$, and if $\ell^{\prime} \in \mathcal{L}$ we delete $\ell$. Otherwise, if none of both is contained in $\mathcal{L}$, we delete that label with higher costs; ties are broken arbitrarily. Afterwards, Assumption 1 is satisfied. Further, for each metro line $C$ of $I$ we iterate through the stops of $C$ from its beginning to its end. Doing so, we delete candidates from $\mathcal{K}$ violating Assumption 2 as described as follows. Let $s$ be the currently considered stop. For each candidate $\ell \in \mathcal{K}_{s}$ we check for each stop $s^{\prime}$ with $s^{\prime}<s$ whether there is a label $\ell^{\prime} \in \mathcal{K}_{s^{\prime}}$ that intersects $\ell$. If $\ell^{\prime}$ exists, we check whether each candidate $\ell^{\prime \prime} \in \mathcal{K}_{s^{\prime \prime}}$ of any stop $s^{\prime \prime}$ with $s^{\prime}<s^{\prime \prime}<s$ intersects $\ell$ or $\ell^{\prime}$. If this is not the case, we delete $\ell$ if $\ell$ is not contained in $\mathcal{L}$, and otherwise we delete $\ell^{\prime}$. Note that not both can be contained in $\mathcal{L}$. By construction each metro line of instance $I$ then satisfies Assumption 2 , where $\mathcal{L} \subseteq \mathcal{K}$.

Finally, we ensure that the metro lines in $\mathcal{M}$ become independent in the sense that no candidates of stops belonging to different metro lines intersect and no candidate intersects any metro line. Hence, 
after this step, the metro lines can independently be labeled such that the resulting labelings compose to a labeling of $I$.

We first rank the candidates of $\mathcal{K}$ as follows. For each metro line $C \in \mathcal{M}$ we construct a labeling $\mathcal{L}_{C}$ using the dynamic program for the two sided case as presented in Section 4 . Due to the previous step, those labelings exist. Note that for two metro lines $C, C^{\prime} \in \mathcal{M}$ there may be labels $\ell \in \mathcal{L}_{C}$ and $\ell^{\prime} \in \mathcal{L}_{C^{\prime}}$ that intersect each other. For each candidate $\ell \in \mathcal{K}$ we set $v_{a l}=1$ if $\ell \in \mathcal{L}_{C}$ for a metro line $C \in \mathcal{M}$, and $v_{a l}=0$ otherwise. A candidate $\ell \in \mathcal{K}$ has a smaller rank than a candidate $\ell^{\prime} \in \mathcal{K}$, if $v a l_{\ell}>v a_{\ell^{\prime}}$ or $v l_{\ell}=v l_{\ell^{\prime}}$ and $w_{1}(\ell) \leq w_{1}\left(\ell^{\prime}\right)$; ties are broken arbitrarily.

We now greedily remove candidates from $\mathcal{K}$ until all metro lines are independent. We create a conflict graph $G=(V, E)$ such that the vertices of $G$ are the candidates and the edges model intersections between candidates, i.e., two vertices are adjacent if and only if the corresponding labels intersect. Then, we delete all vertices whose corresponding labels intersect any metro line. Afterwards, starting with $\mathcal{I}=\emptyset$, we construct an independent set $\mathcal{I}$ on $G$ as follows. First, we add all vertices of $G$ to $\mathcal{I}$, whose labels are contained in $\mathcal{L}$, and delete them and their neighbors from $G$. Since the labels in $\mathcal{L}$ do not intersect, $\mathcal{I}$ is an independent set of the original conflict graph. In the increasing order of their ranks, we remove each vertex $v$ and its neighbors from $G$. Each time we add $v$ to $\mathcal{I}$; obviously sustaining that $\mathcal{I}$ is an independent set in $G$. We then update for each stop $s \in \mathcal{S}$ its candidate set $\mathcal{K}_{s}$ to $\mathcal{K}_{s} \leftarrow\left\{\ell \in \mathcal{K}_{s} \mid\right.$ vertex of $\ell$ is contained in $\left.\mathcal{I}\right\}$. Since all labels of $\mathcal{L}$ are contained in $\mathcal{I}$, there is a labeling for $(\mathcal{M}, \mathcal{S}, \mathcal{K}, w)$ based on the new candidate set $\mathcal{K}$.

4th Step - Final Candidate Selection. Let $I=(\mathcal{M}, \mathcal{S}, \mathcal{K}, w)$ be the instance after applying the third step and $\mathcal{L}$ be the labeling that has been created in the first step. By the previous step the metro lines are in the sense independent that candidates of stops belonging to different metro lines do not intersect. Further, they all satisfy Assumption 1 and Assumption 2 Hence, we use the dynamic programming approach of Section 4 in order to label them independently. The composition of those labelings is then a labeling $\mathcal{L}$ of $I$.

\section{Alternative Approaches}

We now present the three approaches IlpAlg, ScaleAlg, GreedyAlg, which are adaptions of our workflow. We use those to experimentally evaluate our approach against alternatives. While GreedyAlg is a simple and fast greedy algorithm, IlpAlg and ScaleAlg are based on an ILP formulation.

\subsection{Integer Linear Programming Formulation}

To assess the impact of the second phase of our approach, we present an integer linear programming formulation that optimally solves MetroMAPLABELING with respect to the required cost function. Let $(\mathcal{M}, \mathcal{S}, \mathcal{K}, w)$ be an instance of that problem, which we obtain after the first phase of our approach. We first note that we apply a specific cost function (see Section 5). The cost function $w_{3}$, which rates two consecutive switchovers $\sigma=\left(\ell_{1}, \ell_{2}\right)$ and $\sigma^{\prime}=\left(\ell_{1}^{\prime}, \ell_{2}^{\prime}\right)$ of a labeling $\mathcal{L}$, does not rely on the actual switchovers, but only on their positions on the corresponding metro line. Hence, we may assume that $w_{3}$ expects the stops of $\ell_{1}$ and $\ell_{1}^{\prime}$. This assumption helps us reduce the number of variables.

For each candidate $\ell \in \mathcal{K}$ we define a binary variable $x_{\ell} \in\{0,1\}$. If $x_{\ell}=1$, we interpret it such that $\ell$ is selected for the labeling. We introduce the following constraints.

$$
\begin{aligned}
x_{\ell} & =0 & & \forall \ell \in \mathcal{K} \text { that intersect a metro line. } \\
x_{\ell}+x_{\ell^{\prime}} & \leq 1 & & \forall \ell, \ell^{\prime} \in \mathcal{K} \text { that intersect. } \\
\sum_{\ell \in \mathcal{K}_{s}} x_{\ell} & =1 & & \forall s \in S
\end{aligned}
$$


Moreover, for each metro line $C \in \mathcal{M}$ we define the following variables. To that end, let $\mathcal{P} \in \mathcal{K}_{C} \times \mathcal{K}_{C}$ be the set of all consecutive labels and let $s_{1}, \ldots, s_{n}$ be the stops of $C$ in that particular order.

$$
\begin{aligned}
y_{\ell, \ell^{\prime}} & \in\{0,1\}, & & \forall\left(\ell, \ell^{\prime}\right) \in \mathcal{P} . \\
z_{i} & \in\{0,1\}, & & 1 \leq i<n \\
h_{i, j} & \in\{0,1\}, & & 1 \leq i<n \text { and } i<j<n
\end{aligned}
$$

If $y_{\ell, \ell^{\prime}}=1$, we interpret it such that both $\ell$ and $\ell^{\prime}$ are selected for the labeling. If $z_{i}=1$, we interpret it such that the selected labels of the stops $s_{i}$ and $s_{i+1}$ form a switchover. If $h_{i, j}=1$, we interpret it such that the selected labels at $s_{i}, s_{i+1}, s_{j}$ and $s_{j+1}$ form two consecutive switchovers. Further, for each metro line $C$ we introduce the following constraints. In these constraints $L(\mathcal{K})$ denotes the set of labels that lie to the left of $C$, and $R(\mathcal{K})$ denotes the set of labels that lie to the right of $C$.

$$
\begin{array}{rl}
x_{\ell}+x_{\ell^{\prime}}-1 \leq y_{\ell, \ell^{\prime}} & \forall\left(\ell, \ell^{\prime}\right) \in \mathcal{P} \\
\sum_{\ell \in L\left(\mathcal{K}_{s_{i}}\right)} x_{\ell}+\sum_{\ell \in R\left(\mathcal{K}_{s_{i+1}} x_{\ell}-1 \leq z_{i}\right.} & 1 \leq i<n \\
\sum_{\ell \in R\left(\mathcal{K}_{s_{i}}\right)} x_{\ell}+\sum_{\ell \in L\left(\mathcal{K}_{s_{i+1}}\right)} x_{\ell}-1 \leq z_{i} & 1 \leq i<n \\
z_{i}+z_{j}-1-\sum_{i<k<j} z_{k} \leq h_{i, j} & 1 \leq i<j<n
\end{array}
$$

We further define for each metro line $C$ the following linear term.

$$
w(C):=\sum_{\ell \in \mathcal{K}_{C}} x_{\ell} \cdot w_{1}(\ell)+\sum_{\left(\ell, \ell^{\prime}\right) \in \mathcal{P}} y_{\ell, \ell^{\prime}} \cdot w_{2}\left(\ell, \ell^{\prime}\right)+\sum_{\substack{1 \leq i<n \\ i<j<n}} h_{i, j} \cdot w_{3}\left(s_{i}, s_{j}\right)
$$

Subject to the presented Constraints $(3)-(9)$ we then minimize

$$
\sum_{C \in \mathcal{M}} w(C) .
$$

Consider a variable assignment that minimizes (11) and satisfies Constraints (3)- (9). We show that

$$
\mathcal{L}=\left\{\ell \in \mathcal{K} \mid x_{\ell}=1\right\}
$$

is an optimal labeling of the given instance with respect to the given cost function. First of all, $\mathcal{L}$ is a valid labeling. Constraint (3) ensures that no label in $\mathcal{L}$ intersects any metro line. By Constraint (4) the labels in $\mathcal{L}$ are pairwise disjoint. Finally, by Constraint (5) for each stop there is exactly one label contained in $\mathcal{L}$. In particular, for a metro line $C \in \mathcal{M}$, the set $\mathcal{L}_{C}=\mathcal{L} \cap \mathcal{K}_{C}$ is a valid labeling of $C$.

We now show that $w\left(\mathcal{L}_{C}\right)=w(C)$ for any metro line $C \in \mathcal{M}$. Since we minimize (11), this implies the optimality of $\mathcal{L}$. Obviously, for a label $\ell \in \mathcal{K}_{C}$ the cost $w_{1}(\ell)$ is taken into account in $w(C)$ if and only if $\ell$ belongs to $\mathcal{L}$.

By Constraint (6) for two consecutive labels $\ell$ and $\ell^{\prime}$ of $C$ we have $y_{\ell, \ell^{\prime}}=1$ if both are contained in $\mathcal{L}$. Further, by the minimality of (11), if at least one of both labels does not belong to $\mathcal{L}$, it holds $y_{\ell, \ell^{\prime}}=0$. Hence, $w_{2}\left(\ell, \ell^{\prime}\right)$ is taken into account in $w(C)$ if and only if both $\ell$ and $\ell^{\prime}$ belong to $\mathcal{L}$.

By Constraint (7) and Constraint (8) it holds $z_{i, j}=1$ if the labels $\ell_{i} \in \mathcal{L}$ and $\ell_{i+1} \in \mathcal{L}$ of the consecutive stops $s_{i}$ and $s_{i+1}$ form a switchover in $\mathcal{L}$. Hence, by Constraint $(9)$ it further holds $h_{i, j}=1$ if the labels of $s_{i}$ and $s_{i+1}$ as well as $s_{j}$ and $s_{j+1}$ form switchovers $\sigma_{i}$ and $\sigma_{j}$ in $\mathcal{L}$, and, furthermore, there is no other switchover in between $\sigma_{i}$ and $\sigma_{j}$, i.e., both switchovers are consecutive. On the other hand, by the minimality of $w(C)$ in all other cases it holds $h_{i, j}=0$. Hence, $w_{3}\left(s_{i}, s_{j}\right)$ is taken into account in $w(C)$ if and only if $\sigma_{i}$ and $\sigma_{j}$ are consecutive switchovers in $\mathcal{L}$. Altogether we obtain the following theorem. 
Theorem 4. Given an optimal variable assignment for the presented ILP formulation, the set $\mathcal{L}=$ $\left\{\ell \in \mathcal{K} \mid x_{\ell}=1\right\}$ is an optimal labeling of $(\mathcal{M}, \mathcal{S}, \mathcal{K})$ with respect to $w$.

The approach ILPALG simply replaces the second phase of DPALG by that ILP formulation. Hence, it solves the second phase optimally. The approach ScAlEAlg samples a predefined scaling range $\left[x_{\min }, x_{\max }\right]$, which is also used by DpALG. For each scale $x$ it scales the candidates correspondingly. Using the ILP formulation it then checks whether the candidates admit a labeling. Hence, we approximately obtain the greatest scaling factor that admits a labeling.

\subsection{Greedy-Algorithm}

The algorithm GREeDYALG replaces the dynamic programming approach in our workflow as follows. Starting with the solution $\mathcal{L}$ enforced by the 1st step, the greedy algorithm iterates once through the stops of $C$. For each stop $s$ of $C$ it selects the candidate $\ell \in \mathcal{K}_{s}$ that minimizes $w_{1}(\ell)+w_{2}\left(\ell_{p}, \ell\right)+w_{2}\left(\ell, \ell_{s}\right)$ among all valid candidates in $\mathcal{K}_{s}$, where $\ell_{p} \in \mathcal{L}$ is the candidate selected for the previous stop $s_{p}$ and $\ell_{s} \in \mathcal{L}$ is the candidate for the successive stop. It replaces the candidate of $s$ in $\mathcal{L}$ with $\ell$.

\section{Evaluation}

To evaluate our approach presented in Section 6, we did a case study on the metro systems of Sydney (173 stops) and Vienna (84 stops), which have been used as benchmarks before 511114. For Sydney we took the curved layout from [5, Fig. 1a] and the octilinear layouts from [11, Fig. 9a,b] [14, Fig. 10.], while for Vienna we took the curved layout from [5, Fig. 8c] and the ocitlinear layouts from [11, Fig. 13a,b]. See also Table 1 for an overview of the instances. Since the metro lines of Sydney are not only paths, we disassembled those metro lines into single paths. We did this by hand and tried to extract as long paths as possible. Hence, the instances of Sydney decompose into 12 lines and the instances of Vienna into 5 lines. We took the positions of the stops as presented in the corresponding papers. In the curved layout of Sydney we removed the stops Tempe and Martin Place (in Fig. 9(a) marked as red dots), because both stops are tightly enclosed by metro lines such that only the placement of very small labels is possible. This is not so much a problem of our approach, but of the given layout. In a semi-automatic approach the designer would then need to change the layout. For the curvilinear layouts we used labels of CurvedStyle and for the octilinear layouts we used labels of OctilinStyle. For the layouts of Sydney2, Sydney3 and Vienna2 the authors present labelings; see Fig. 10, Fig. 11. and Fig. 12. For any other layout they do not present labelings.

Table 1: Overview of considered instances. Style: Style of map and applied labels. $\frac{s}{s_{\max }}$ : Ratio of applied scale factors. The scale $s_{\max }$ is a lower bound for the largest possible scaling factor (obtained by SCALEALG), and $s$ is the scale computed by the first phase of our workflow.

\begin{tabular}{cccccccc}
\hline Instance & Sydney1 & Sydney2 & Sydney3 & Sydney4 & Vienna1 & Vienna2 & Vienna3 \\
\hline Style & Octi. & Octi. & Octi. & Curved & Octi. & Octi. & Curved. \\
$\frac{s}{s_{\max }}$ & 0.69 & 0.57 & 0.57 & 0.67 & 0.59 & 0.81 & 0.54 \\
Reference & [11, Fig. 9a] & [11, Fig. 9b] & 14, & Fig. 10.] & 5, Fig. 1a] & 11, Fig. 13a] [11, Fig. 13b] & [5, Fig. 8c] \\
\hline
\end{tabular}

The experiments were performed on a single core of an Intel(R) Core(TM) i7-3520M CPU processor. The machine is clocked at $2.9 \mathrm{GHz}$, and has $4096 \mathrm{MB}$ RAM. Our implementation is written in Java. For each instance and each algorithm we conducted 100 runs and took the average running times. Each time before we started the 100 runs, we performed 50 runs without measuring the running time in order to warm up the virtual machine (Java(TM) SE Runtime Environment, build 1.8.0_60-b27, 
Table 2: Running times in seconds of the workflow broken down into its two phases and their single steps (if applicable). Algo.: The applied algorithm. Times less than 0.01 seconds are marked with $\star$.

\begin{tabular}{|c|c|c|c|c|c|c|c|c|}
\hline \multirow{2}{*}{\multicolumn{2}{|c|}{ Instance Layout }} & \multirow[t]{2}{*}{ Algo. } & \multicolumn{5}{|c|}{ Phase 1: Creation Phase 2: Selection } & \multirow[b]{2}{*}{ Total } \\
\hline & & & 1 st $2 n d$ & $\sum$ & $1 s t$ & 2nd & $\sum$ & \\
\hline \multirow{4}{*}{ Sydney 1} & \multirow{4}{*}{ Octi. } & DPALG & 0.030 .18 & 0.21 & 0.09 & 0.1 & 0.18 & 0.39 \\
\hline & & GREEDYAlg & 0.020 .18 & 0.2 & $\star$ & $\star$ & $\star$ & 0.2 \\
\hline & & SCALEALG & $0.02-$ & 0.02 & - & - & 69.09 & 69.11 \\
\hline & & ILPALG & 0.020 .17 & 0.19 & - & - & 25.43 & 25.62 \\
\hline \multirow{4}{*}{ Sydney2 } & \multirow{4}{*}{ Octi. } & DPALG & 0.030 .28 & 0.31 & 0.07 & 0.09 & 0.15 & 0.46 \\
\hline & & GreEdyAlG & 0.020 .28 & 0.3 & $\star$ & $\star$ & $\star$ & 0.3 \\
\hline & & SCALEALG & $0.02-$ & 0.02 & - & - & 17.29 & 17.31 \\
\hline & & ILPALG & 0.020 .28 & 0.29 & - & - & 15.17 & 15.46 \\
\hline \multirow{4}{*}{ Sydney3 } & \multirow{4}{*}{ Octi. } & DPALG & 0.030 .17 & 0.2 & 0.07 & 0.09 & 0.16 & 0.36 \\
\hline & & GreEdyAlg & 0.020 .16 & 0.17 & $\star$ & $\star$ & $\star$ & 0.17 \\
\hline & & SCALEALG & $0.02-$ & 0.02 & - & - & 33.61 & 33.63 \\
\hline & & ILPALG & 0.020 .15 & 0.17 & - & - & 21.56 & 21.73 \\
\hline \multirow{4}{*}{ Sydney 4} & \multirow{4}{*}{ Curved } & DPALG & 0.030 .33 & 0.36 & 0.1 & 0.1 & 0.2 & 0.56 \\
\hline & & GreEDyAlG & 0.030 .33 & 0.35 & $\star$ & $\star$ & $\star$ & 0.35 \\
\hline & & SCALEALG & $0.03-$ & 0.05 & - & - & 243.58 & 243.63 \\
\hline & & ILPALG & 0.020 .34 & 0.36 & - & - & 98.81 & 99.17 \\
\hline \multirow{4}{*}{ Vienna1 } & \multirow{4}{*}{ Octi. } & DPAlG & 0.010 .06 & 0.07 & 0.01 & $\star$ & 0.02 & 0.09 \\
\hline & & GreEdyAlG & 0.010 .06 & 0.07 & $\star$ & $\star$ & $\star$ & 0.07 \\
\hline & & SCALEALG & $0.01-$ & 0.01 & - & - & 3.31 & 3.32 \\
\hline & & ILPALG & 0.010 .06 & 0.08 & - & - & 0.55 & 0.63 \\
\hline \multirow{4}{*}{ Vienna2 } & \multirow{4}{*}{ Octi. } & DPALG & 0.010 .09 & 0.1 & $\star$ & $\star$ & $\star$ & 0.11 \\
\hline & & GreEdyAlg & $0.01 \quad 0.1$ & 0.11 & $\star$ & $\star$ & $\star$ & 0.11 \\
\hline & & SCALEAlG & $0.01-$ & 0.01 & - & - & 4.65 & 4.66 \\
\hline & & ILPALG & 0.010 .08 & 0.09 & - & - & 0.36 & 0.45 \\
\hline \multirow{4}{*}{\multicolumn{2}{|c|}{ Vienna3 Curved }} & DPAlg & 0.020 .15 & 0.17 & 0.02 & 0.04 & 0.06 & 0.23 \\
\hline & & GreedyAlg & 0.020 .14 & 0.16 & $\star$ & $\star$ & $\star$ & 0.16 \\
\hline & & SCALEALG & $0.02-$ & 0.02 & - & - & 7.27 & 7.29 \\
\hline & & ILPALG & 0.010 .13 & 0.15 & - & - & 0.49 & 0.64 \\
\hline
\end{tabular}


Table 3: Experimental results for Sydney and Vienna. Algo.: The applied algorithm. Candidates: Values concerning candidates; No. of candidates after the first and third step, $A$ 피 $=$ No. of labels removed to establish Assumption 1 and Assumption 2, $\mathrm{SO}=$ No. of switchovers. Cost: Ratio of costs; $\mathcal{L}_{A}=$ labeling obtained by procedure $A \in\{$ DpAlg, GreedyAlg, IlpAlg, ScaleAlg $\}, \mathcal{L}=$ labeling obtained by procedure ILPAlg. Sequence: Values concerning sequences of labels lying on the same side of their metro line; $\min =$ length of shortest sequence, $\max =$ length of longest sequence, avg.=average length of sequences.

\begin{tabular}{|c|c|c|c|c|c|c|c|c|c|c|c|c|}
\hline & \multirow[b]{2}{*}{ Algo. } & \multicolumn{4}{|c|}{ Candidates } & \multicolumn{4}{|c|}{ Cost } & \multicolumn{3}{|c|}{ Sequence } \\
\hline Instance & & 1 st & $3 \mathrm{rd}$ & A12 & $\mathrm{SO}$ & $\frac{w_{1}\left(\mathcal{L}_{A}\right)}{w_{1}(\mathcal{L})}$ & $\frac{w_{2}\left(\mathcal{L}_{A}\right)}{w_{2}(\mathcal{L})}$ & $\frac{w_{3}\left(\mathcal{L}_{A}\right)}{w_{3}(\mathcal{L})}$ & $\frac{w\left(\mathcal{L}_{A}\right)}{w(\mathcal{L})}$ & $\min$ & $\max$ & avg. \\
\hline \multirow{4}{*}{ Sydney1 } & DPAlg & 1164 & 1004 & 9 & 4 & 1.73 & 1.12 & 0.63 & 1.16 & 3 & 41 & 9.53 \\
\hline & GreedyAlg & 1164 & 1005 & 9 & 15 & 1.73 & 1.87 & 7.62 & 2.64 & 1 & 24 & 5.81 \\
\hline & SCALEAlG & 1164 & - & - & 13 & 2.46 & 1.67 & 2.91 & 1.97 & 3 & 20 & 6.97 \\
\hline & ILPALG & 1164 & - & - & 7 & 1 & 1 & 1 & 1 & 3 & 20 & 8.1 \\
\hline \multirow{4}{*}{ Sydney 2} & DPAlg & 1104 & 963 & 3 & 6 & 1.36 & 1.08 & 3.25 & 1.33 & 1 & 26 & 9.34 \\
\hline & GreedyAlg & 1104 & 964 & 3 & 19 & 2.46 & 1.83 & 19.52 & 3.54 & 1 & 35 & 5.94 \\
\hline & SCALEAlg & 1104 & - & - & 15 & 3.91 & 1.92 & 8.88 & 2.91 & 1 & 19 & 6.76 \\
\hline & ILPALG & 1104 & - & - & 5 & 1 & 1 & 1 & 1 & 3 & 25 & 9.48 \\
\hline \multirow{4}{*}{ Sydney3 } & DPAlg & 1132 & 1013 & 0 & 6 & 1 & 1 & 1 & 1 & 3 & 25 & 8.76 \\
\hline & GreedyAlg & 1132 & 1013 & 0 & 14 & 2.15 & 1.79 & 5.99 & 2.61 & 1 & 23 & 6.9 \\
\hline & SCALEALG & 1132 & - & - & 12 & 2.72 & 2.68 & 3.06 & 2.76 & 2 & 21 & 7.02 \\
\hline & ILPALG & 1132 & - & - & 6 & 1 & 1 & 1 & 1 & 3 & 25 & 8.76 \\
\hline \multirow{4}{*}{ Sydney4 Curved } & DPAlg & 1275 & 1020 & 2 & 8 & 1 & 1.13 & 1.15 & 1.01 & 3 & 27 & 7.38 \\
\hline & GreEDyAlg & 1275 & 1021 & 2 & 28 & 1 & 1.08 & 12.81 & 1.16 & 1 & 24 & 4.82 \\
\hline & SCALEAlg & 1275 & - & - & 13 & 1.05 & 1.69 & 2.28 & 1.1 & 2 & 20 & 6.21 \\
\hline & ILPALG & 1275 & - & - & 7 & 1 & 1 & 1 & 1 & 3 & 27 & 7.92 \\
\hline \multirow{4}{*}{ Vienna1 Octi. } & DPAlG & 466 & 403 & 0 & 2 & 0.74 & 1.29 & 0.99 & 1.07 & 8 & 21 & 12.8 \\
\hline & GREeDYAlG & 466 & 403 & 0 & 7 & 1.26 & 2.43 & 8.59 & 2.48 & 1 & 21 & 9.41 \\
\hline & SCALEALG & 466 & - & - & 6 & 2.04 & 3.14 & 6.22 & 2.99 & 1 & 19 & 10.05 \\
\hline & ILPALG & 466 & - & - & 2 & 1 & 1 & 1 & 1 & 8 & 21 & 12.8 \\
\hline \multirow{4}{*}{ Vienna2 Octi. } & DPALG & 468 & 324 & 1 & 8 & 2.63 & 1.03 & 4.01 & 1.52 & 1 & 16 & 8.12 \\
\hline & GreedyAlg & 468 & 324 & 1 & 18 & 4.79 & 1.72 & 14.23 & 3.36 & 1 & 12 & 4.31 \\
\hline & SCALEALG & 468 & - & - & 6 & 2.63 & 1.07 & 2.55 & 1.4 & 1 & 12 & 7.87 \\
\hline & ILPALG & 468 & - & - & 4 & 1 & 1 & 1 & 1 & 5 & 16 & 10 \\
\hline \multirow{4}{*}{ Vienna3 Curved } & DPAlG & 606 & 535 & 0 & 7 & 1 & 1 & 1 & 1 & 2 & 22 & 10.7 \\
\hline & GREEDYAlG & 606 & 535 & 0 & 11 & 0.98 & 1.47 & 1.92 & 1.06 & 1 & 17 & 6.6 \\
\hline & SCALEALG & 606 & - & - & 13 & 1.18 & 3.2 & 2.18 & 1.34 & 1 & 16 & 7.43 \\
\hline & ILPALG & 606 & - & - & 7 & 1 & 1 & 1 & 1 & 2 & 22 & 10.7 \\
\hline
\end{tabular}




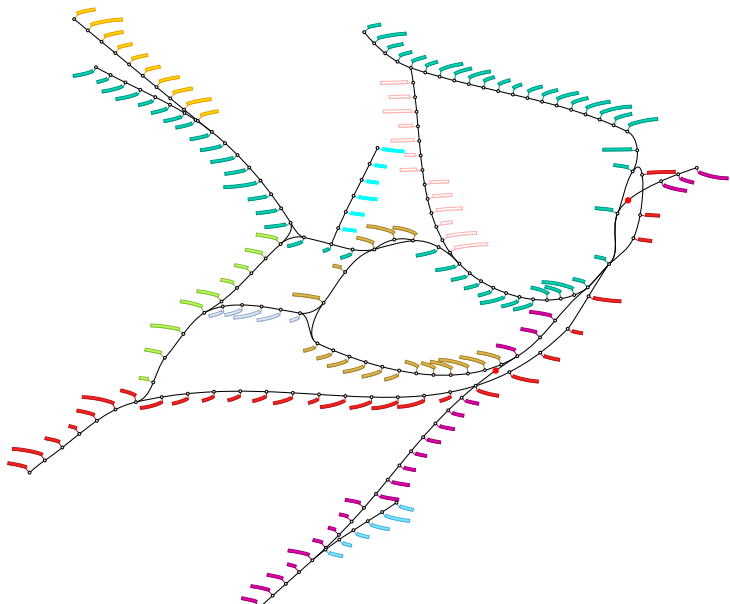

(a) Sydney4: DpAlg, CurvedStyle Layout from [5, Fig. 1a.].

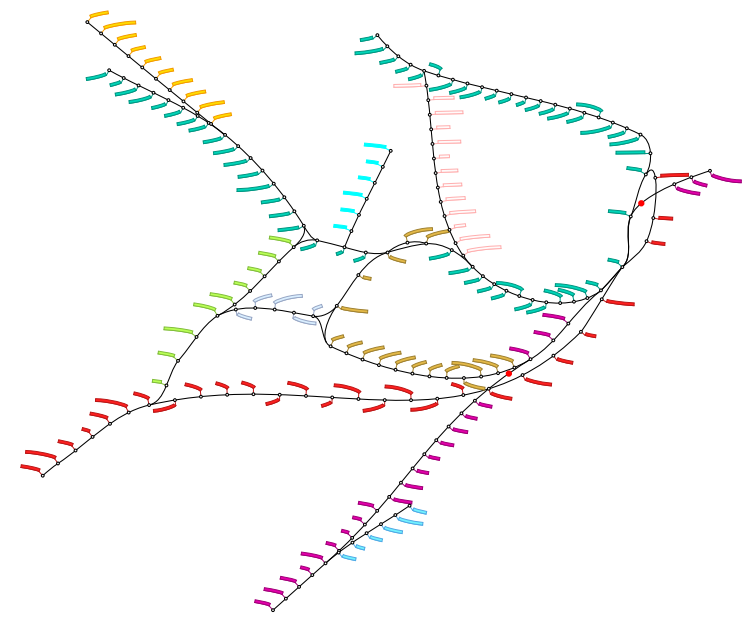

(b) Sydney4: GreedyAlg, CurvedStyle Layout from [5, Fig. 1a].

Fig. 9: Labelings for Sydney using DpAlg and GreedyAlg.

Oracle). We did this in order to measure the actual running times of our algorithms and not to measure the time that the virtual machine of Java spends for loading classes and optimizing byte code.

Table 2 and Table 3 present our quantitative results for the considered instances. For Sydney 4 labelings are found in Fig. 9 and for Sydney2, Sydney3 and Vienna2 labelings created by DpALG are found in Fig. 10, Fig. 11, Fig. 12, respectively. The labelings of all instances are found in the appendix.

We first note that with respect to the total number of created candidates only few labels are removed for enforcing Assumption 1 and Assumption 2 see Table 3, A 팡. This indicates that requiring those assumptions is not a real restriction on a realistic set of candidates, even though they seem to be artificial.

Running time. Even for large networks as Sydney, our algorithm DpALG needs less than 0.6 seconds; see Table 2. This shows that our approach is applicable for scenarios in which the map designer wants to adapt the layout and its labeling interactively. In particular in those scenarios not every of the four steps must be repeated each time, which improves computing time. For example, after once applying the scaling step (1st phase, 2nd step - the most time consuming step), the instance does not need to be rescaled again, but the relation between label size and map size is determined. Further, DpAlg is only moderately slower than GreEdyAlg; 0.21 seconds in maximum, see Table 2 , Sydney4. On the other hand, the approaches IlpAlg and ScaleAlg are not alternatives, because their running times are much worse; over 1 minute in maximum; see Table 2, Sydney4.

Quality. We observe that in all labelings created by DPALG there are only few switchovers, namely 4-8; see Table 3, column SO. Hence, there are long sequences of consecutive labels that lie on the same side of their metro line; see corresponding figures and Table 3, column Sequence. Together with the ILP based approach ILPALG, it yields the solution with the longest sequences in average. In particular the switchovers are placed such that those sequences are regularly sized. The labels of a single sequence are mostly directed into the same $x$-direction and in particular they are similarly shaped so that those sequences of labels form regular patterns as desired. The alignment of the labels is chosen so that they blend in with the alignment of their adjacent labels. In comparison with the solution of ILPALG, the costs of DpAlg never exceed a factor of 1.52; see Table 3 , column $\frac{w\left(\mathcal{L}_{\text {DPALG }}\right)}{w(\mathcal{L})}$. For the instances Sydney 4 and Vienna3 it even obtains a solution with the same costs. For the other instances, DpALg basically spends its additional costs on the choice of the single labels $\left(\frac{w_{1}\left(\mathcal{L}_{\mathrm{DPALG}}\right)}{w_{1}(\mathcal{L})}\right)$ and the distance of switchovers $\left(\frac{w_{3}\left(\mathcal{L}_{\text {DPALG }}\right)}{w_{3}(\mathcal{L})}\right)$. 


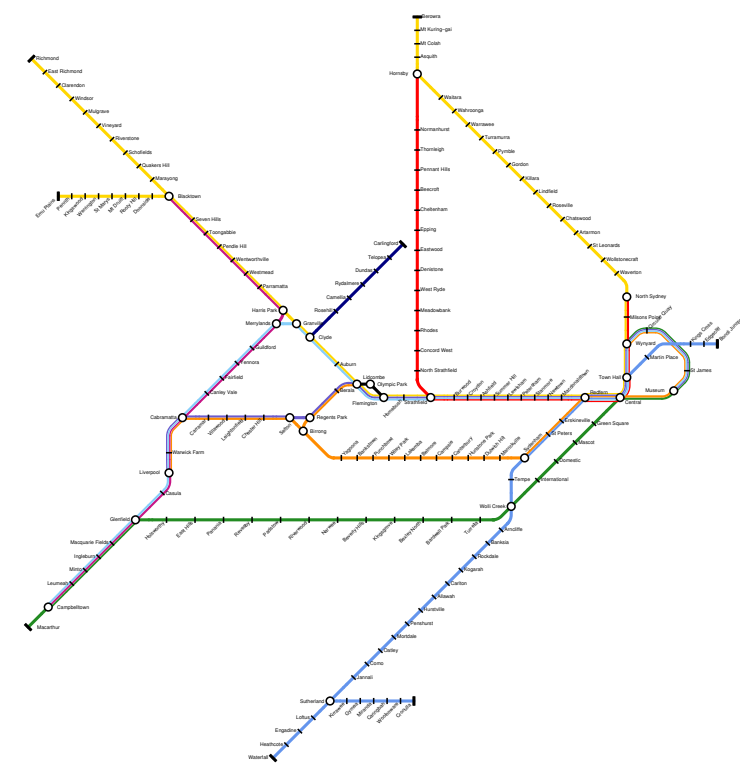

(a) Original labeling by 11

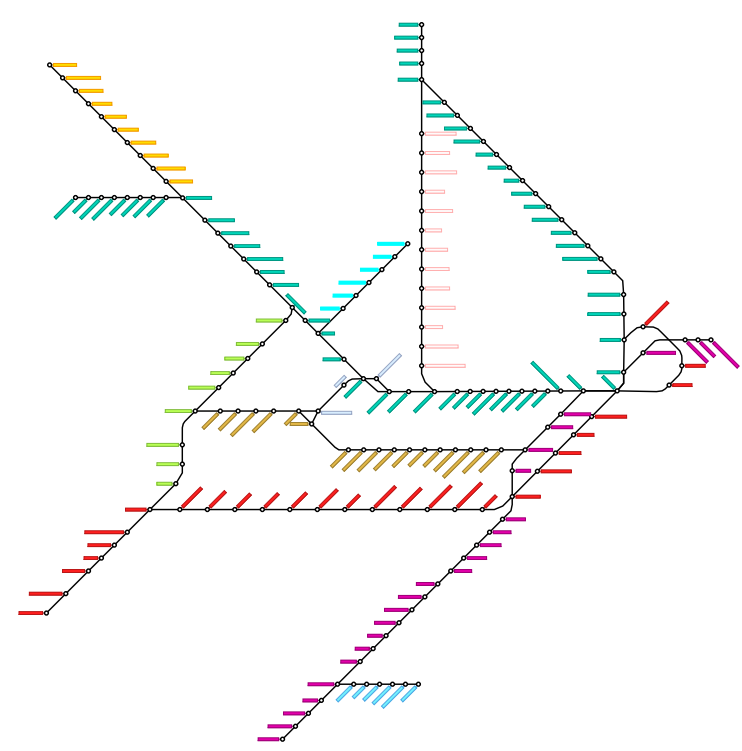

(b) Sydney2: DpAlg, OctilinStyle Layout from [11, Fig. 9b.].

Fig. 10: Labelings for Sydney.

In contrast, GREEDYAlg yields significantly more switchovers; in maximum 20 switchovers more than DpAlg, see Sydney4 . Consequently, there are many distracting switches of labels from one side to the other of the metro line; e.g. see Fig. 9. Although the sequences of consecutive labels lying on the same side may be longer in maximum compared to DPALG, they are much shorter in average; see Table 3 , column Sequence. Further, several adjacent labels point in opposite $x$-directions, which results in distracting effects; see corresponding figures. Altogether, the labelings that are obtained by GreedyAlg do not look regular, but cluttered. DPAlg solves those problems since it considers the metro line globally yielding an optimal labeling for a single line. This observation is also reflected in Table 3 column $\frac{w\left(\mathcal{L}_{\text {DPALG }}\right)}{w(\mathcal{L})}$, which shows that the costs computed by GREEDYALG are significantly larger than the costs computed by DPALG. In particular costs for positioning the switchovers are much worse; Sydney2: $w_{3}\left(\mathcal{L}_{\text {GrembYALG }}\right) / w_{3}(\mathcal{L})=19.52, w_{3}\left(\mathcal{L}_{\text {DPALG }}\right) / w_{3}(\mathcal{L})=3.25$ and Vienna2: $w_{3}\left(\mathcal{L}_{\text {GrebDYALG }}\right) / w_{3}(\mathcal{L})=14.23$, $w_{3}\left(\mathcal{L}_{\mathrm{DPALG}}\right) / w_{3}(\mathcal{L})=4.01$. Hence, the better quality of DPALG prevails the slightly better running time of GREedyAlg.

Concerning the computed scale factor in the first phase of DPALG, the labels are smaller than those produced by SCALEAlg by a factor of $0.54-0.81$; see Table 1 . While this seems to be a drawback on the first sight, the smaller size provides necessary space that is used to obtain a labeling of higher quality with respect to the number and the placement of switchovers. Hence, the solutions of SCALEALG have more switchovers (except for Vienna2) and shorter sequences of labels lying on the same side in average than DpAlg; see Table 3, column SO and Sequence.

We observe that both Nöllenburg and Wolff's and our labelings of Sydney look quite similar, whereas our labeling has less switchovers; see Fig. 10. The same applies for the labelings of the layout of Vienna; see Fig. 12, Recall that their approach needed more than 10 hours to compute a labeled metro map of Sydney. Since they need only up to 23 minutes to compute the layout without labeling, it lends itself to first apply their approach to gain a layout and then to apply our approach to construct a corresponding labeling.

Wang and Chi present in their paper [14 an approach that is divided into two phases. In the first phase they compute the layout of the metro map and then in the second phase they create a labeling for 


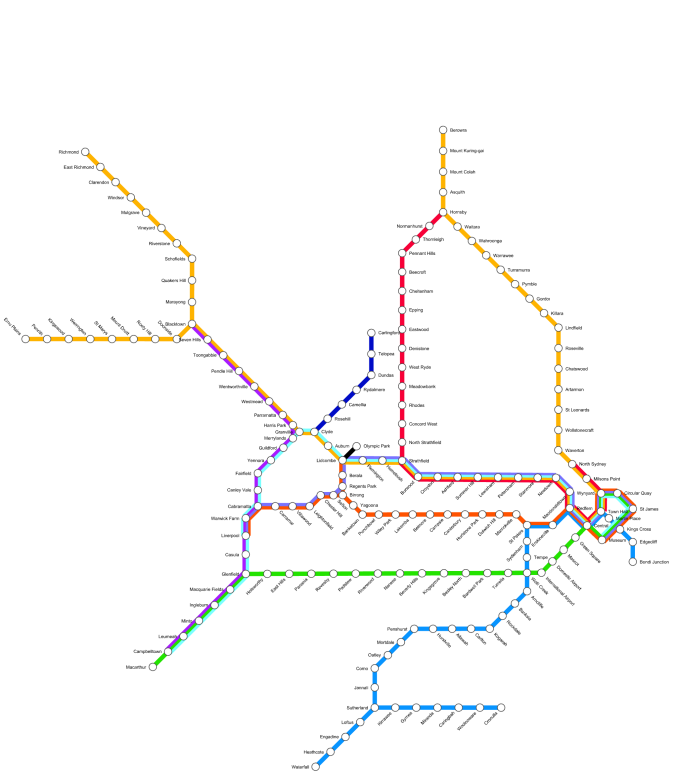

(a)

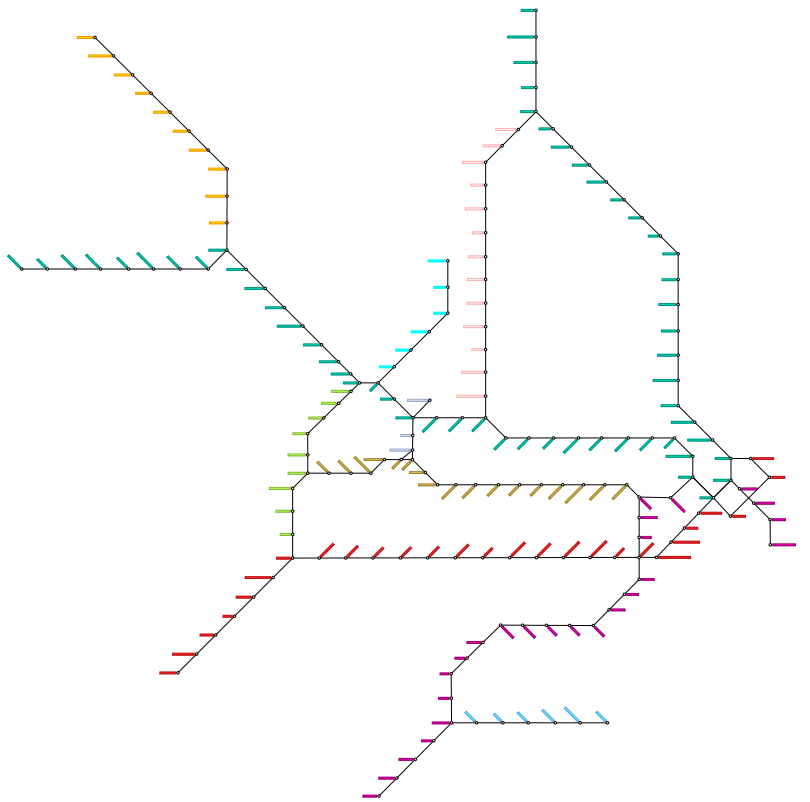

(b)

Fig. 11: Labelings for Sydney. (a) Original Labeling presented by Wang and Chi [14] (b) Sydney3: DPAlg.

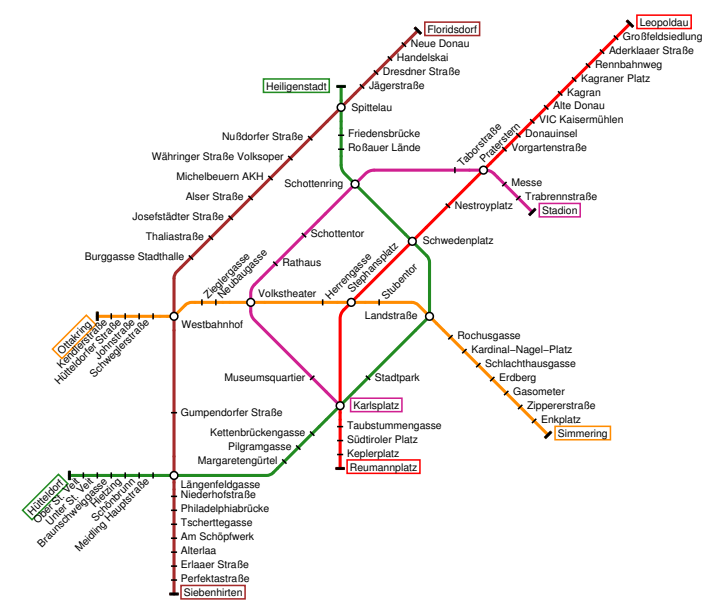

(a)

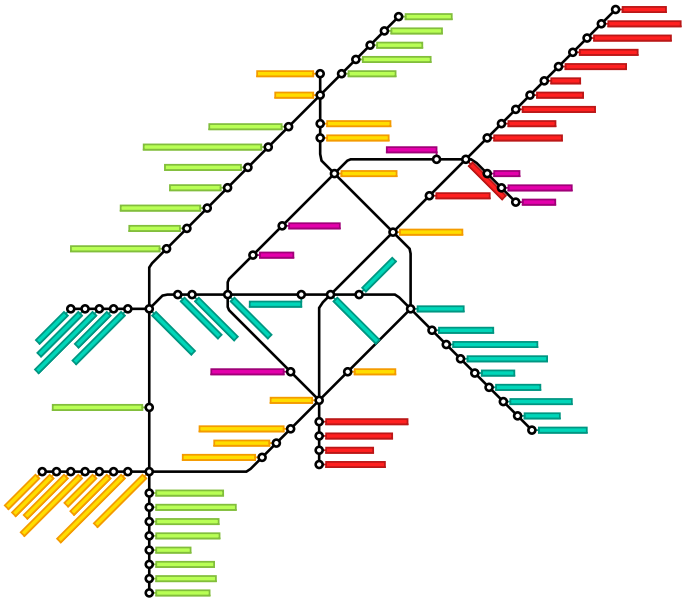

(b)

Fig. 12: Labelings for Vienna. (a) Original Labeling presented by Nöllenburg and Wolff [1] (b) Vienna2: DPAlg.

that layout. For both steps they formulate energy functions expressing their desired objectives, which then are locally optimized. Figure 11(a) shows the metro map of Sydney created by their approach. In comparison, Fig. 11(b) shows the same layout with a labeling created by our approach. Both labelings look quite similar. While our approach needed 0.26s (see Table 2, Sydney3), their approach needed less than 0.1s on their machine. However, their approach does not guarantee that the labels are occlusionfree, but labels may overlap with metro lines and other labels. This may result in illegible drawings 


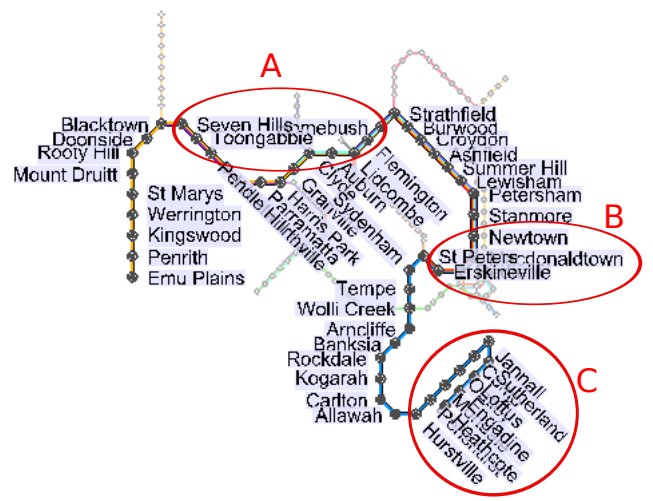

(a)

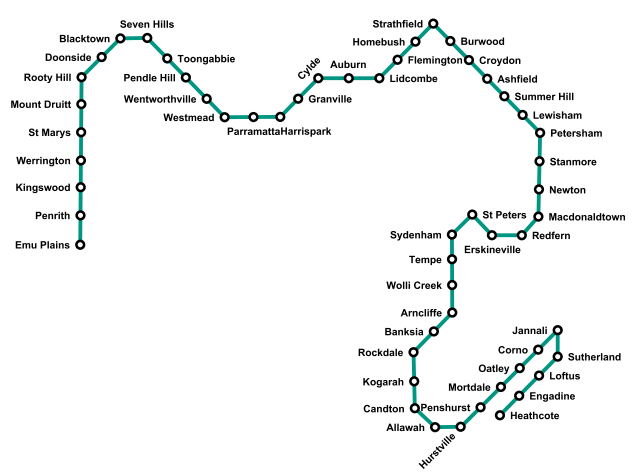

(b)

Fig. 13: Comparison of two labelings for the same line. (a) Labeling is created by the tool presented by Wang and Chi 14. (b) Labeling is created by DpAlg.

of metro maps. For example Fig. 13 shows two labelings of a metro line of Sydney that has been laid out by the tool of Wang and Chi. Figure 13(a) shows a labeling that has been created by their tool, while Fig. 13(b) shows a labeling that has been created by our approach. The labeling of Wang and Chi has several serious defects that makes the map hardly readable. The marked regions A, B and C show labels that overlap each other. Hence, some of the labels are obscured partly, while some of the labels are completely covered by other labels. For example in region B the label St. Peters and the label Erskinville overlap the label Macdonaldtown such that it is hardly viewable. Further, region C contains two diagonal rows of stops aligned parallel. While the upper row is visible, the lower row is almost completely covered by labels. Further, the labels of the upper row obscure the labels of the lower row. In contrast our approach yields an occlusion-free labeling, such that each label and each stop is easily legible. We therefore think that our approach is a reasonable alternative for the labeling step of Wang and Chi's approach. In particular, we think that the better quality of our approach prevails the better running time of Wang and Chi's approach.

In conclusion our workflow is a reasonable alternative and improvement for the approaches presented both by Nöllenburg and Wolff, and by Wang and Chi. In the former case, our approach is significantly faster, while in contrast to the latter case we can guarantee occlusion-free labelings.

Acknowledgment. We sincerely thank Herman Haverkort, Arlind Nocaj, Aidan Slingsby and Jo Wood for helpful and interesting discussions.

\section{References}

1. P. K. Agarwal, M. van Kreveld, and S. Suri. Label placement by maximum independent set in rectangles. Comp. Geom.-Theor. Appl., 11:209-218, 1998. 2

2. B. Aspvall, M. F. Plass, and R. E. Tarjan. A linear-time algorithm for testing the truth of certain quantified boolean formulas. Inf. Process. Lett., 8(3):121-123, 1979. 16

3. J. Christensen, J. Marks, and S. Shieber. An empirical study of algorithms for point-feature label placement. Acm. T. Graphic., 14(3):203-232, 1995. 2

4. T. H. Cormen, C. E. Leiserson, R. L. Rivest, and C. Stein. Introduction to Algorithms (3. Edition). MIT Press, 2009. 11

5. M. Fink, H. Haverkort, M. Nöllenburg, M. Roberts, J. Schuhmann, and A. Wolff. Drawing Metro Maps Using Bézier Curves. In W. Didimo and M. Patrignani, editors, Graph Drawing, volume 7704 of LNCS, pages 463-474. Springer Berlin Heidelberg, 2013. 1, 19,22 
6. M. Formann and F. Wagner. A packing problem with applications to lettering of maps. In ACM Sympos. on Comput. Geom., pages 281-288, 1991. 2, 4

7. R. J. Fowler, M. S. Paterson, and S. L. Tanimoto. Optimal packing and covering in the plane are npcomplete. Inf. Process. Lett., 12(3):133 - 137, 1981. 4

8. E. Imhof. Positioning names on maps. Am. Cartographer, pages 128-144, 1975. 1, 2, $3,9,14$

9. K. G. Kakoulis and I. G. Tollis. A unified approach to labeling graphical features. In Proceedings of the Fourteenth Annual Symposium on Computational Geometry, SCG '98, pages 347-356, New York, NY, USA, 1998. ACM. 2

10. D. Lichtenstein. Planar formulae and their uses. SIAM J. Comput., 11(2):329-343, 1982 . 4.5

11. M. Nöllenburg and A. Wolff. Drawing and labeling high-quality metro maps by mixed-integer programming. IEEE T. Vis. Comput. Gr., 17(5):626-641, 2011. 1, 19, 23, 24

12. J. Stott, P. Rodgers, J. Martinez-Ovando, and S. Walker. Automatic metro map layout using multicriteria optimization. IEEE T. Vis. Comput. Gr., 17(1):101-114, 2011. 1

13. A. van Goethem, W. Meulemans, A. Reimer, H. Haverkort, and B. Speckmann. Topologically safe curved schematisation. Cartogr. J., 50(3):276-285, 2013. 1 .

14. Y.-S. Wang and M.-T. Chi. Focus+context metro maps. IEEE T. Vis. Comput. Gr., 17(12):2528-2535, 2011. 1, 19, 23, 24, 25

15. A. Wolff. Graph drawing and cartography. In R. Tamassia, editor, Handbook of Graph Drawing and Visualization, chapter 23, pages 697-736. CRC Press, 2013. 1 


\section{A Labelings}

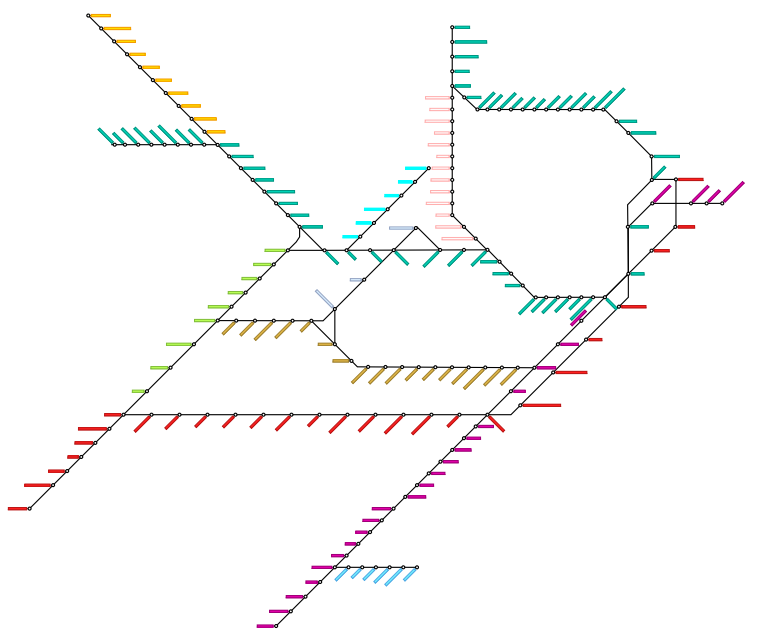

(a) DpAlg

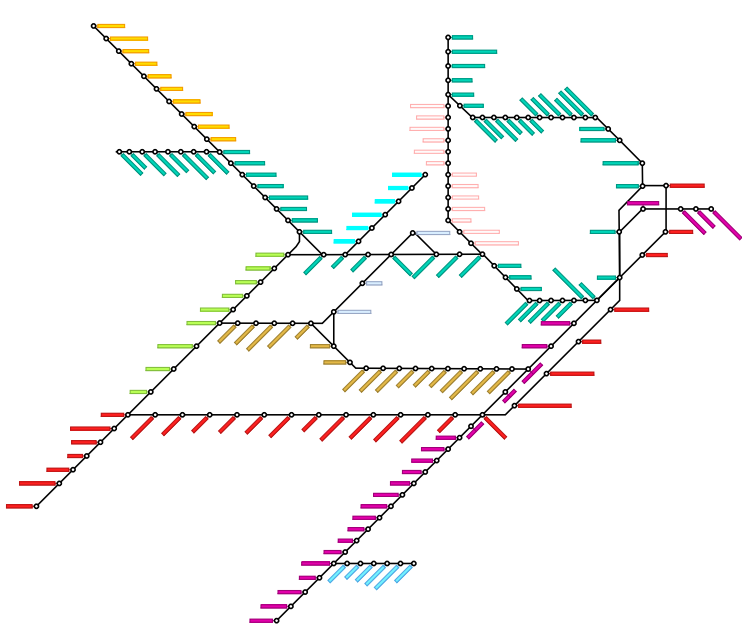

(c) ScaleAlg

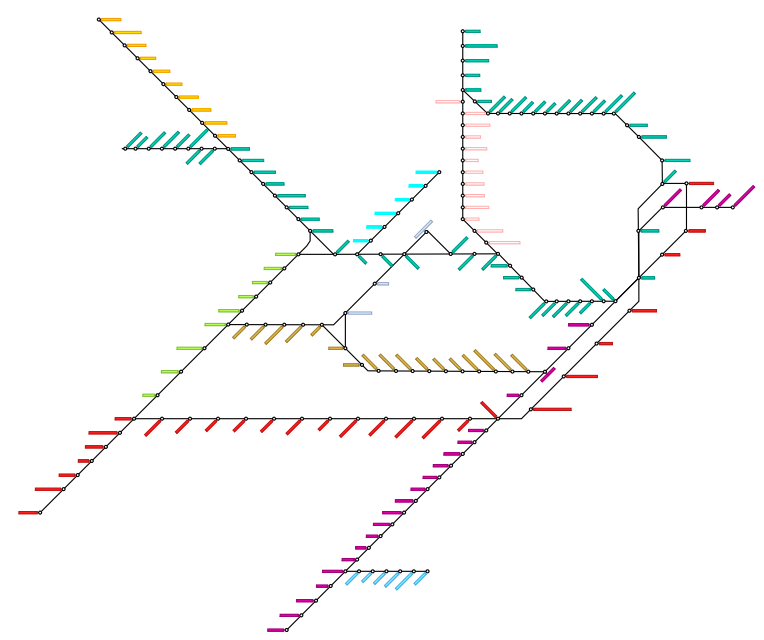

(b) GreedyAlg

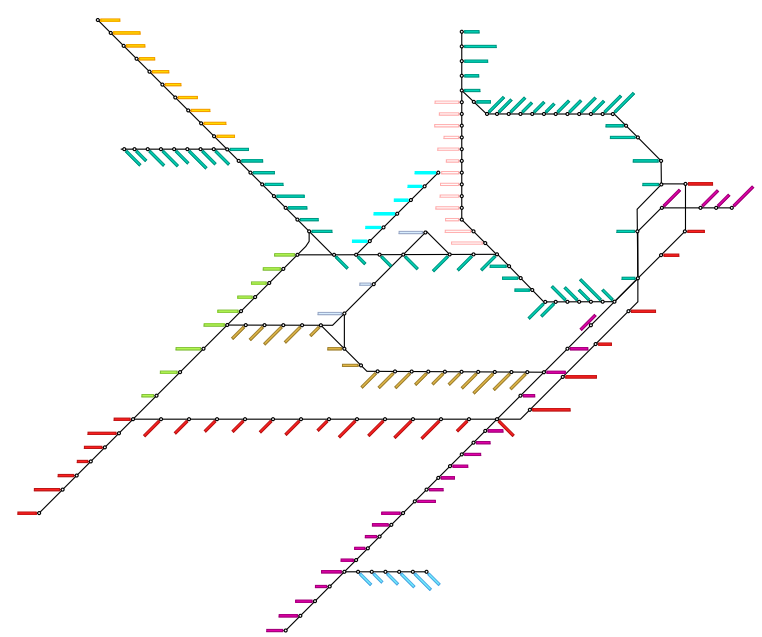

(d) ILPALG

Fig. 14: Labelings for instance Sydney1. 


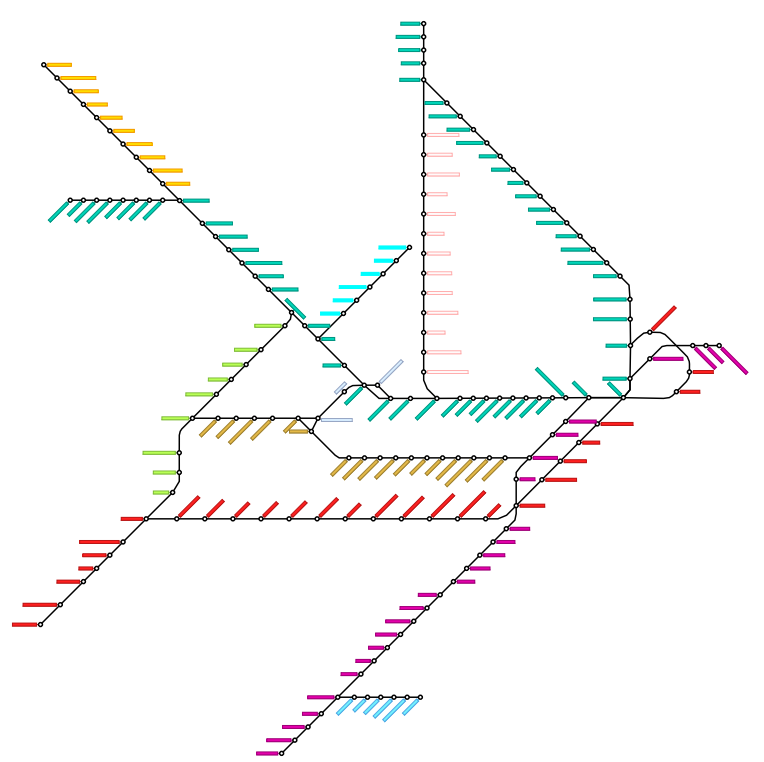

(a) DpAlg

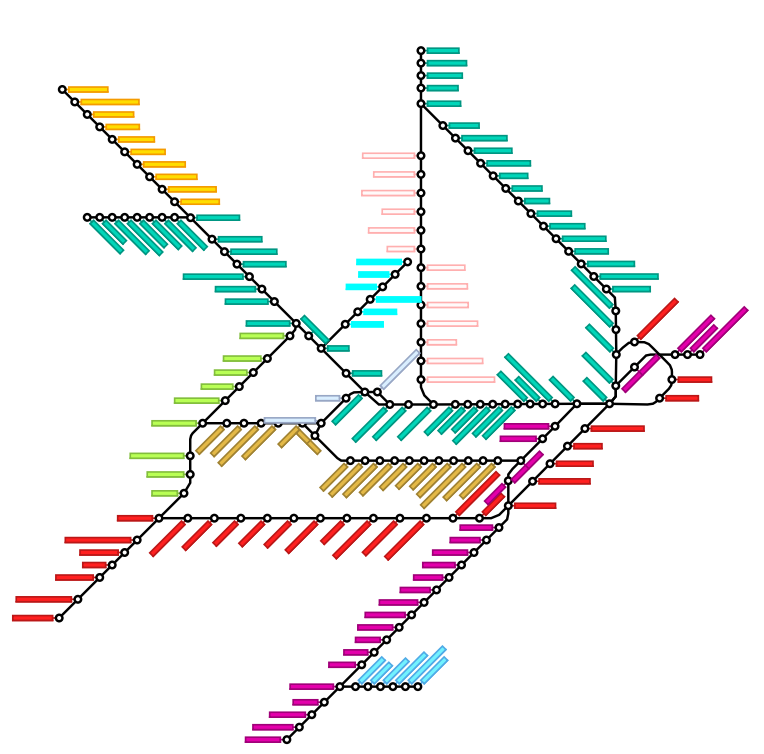

(c) ScaleAlg

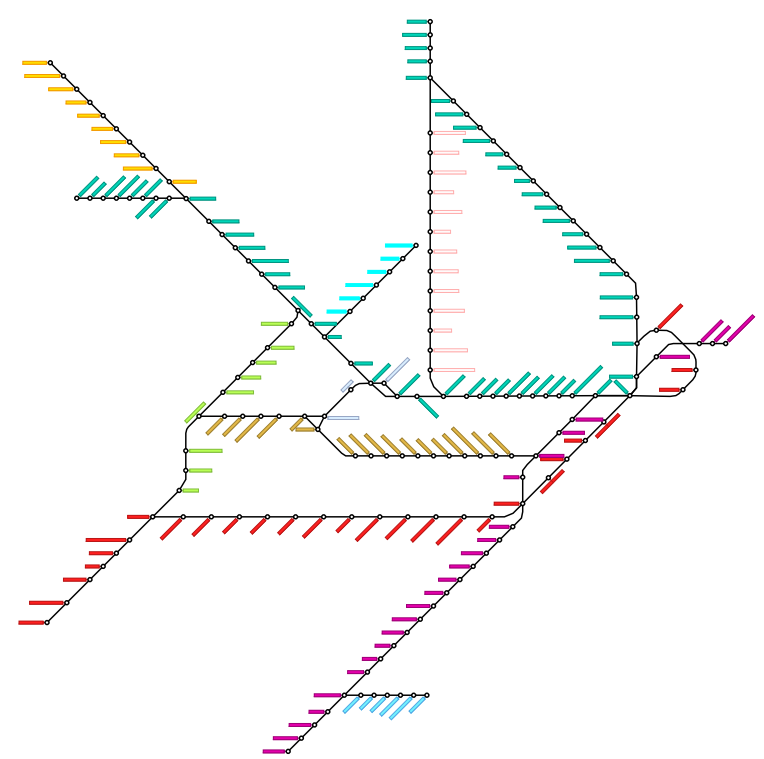

(b) GreedyAlg

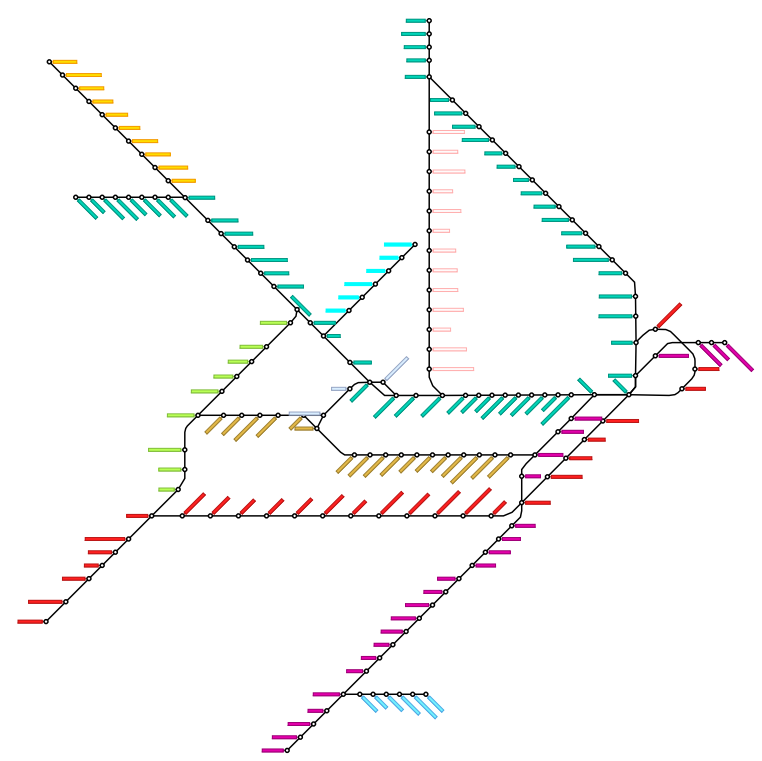

(d) ILPALG

Fig. 15: Labelings for instance Sydney2. 


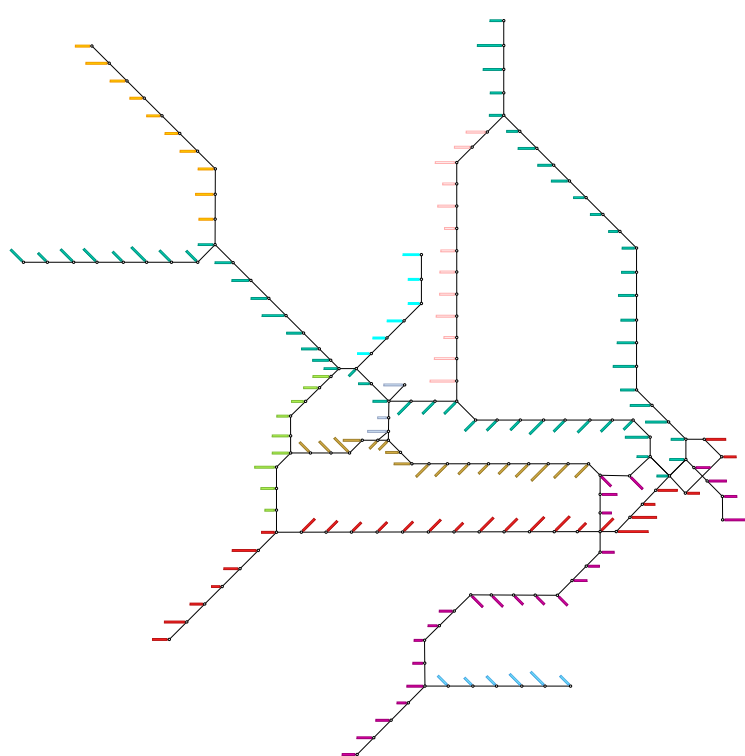

(a) DpAlg

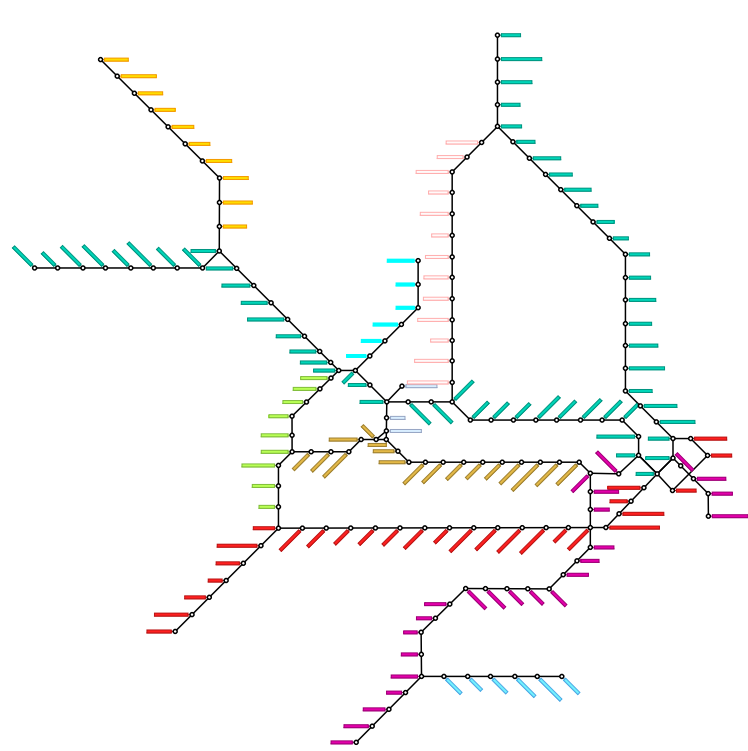

(c) ScaleAlg

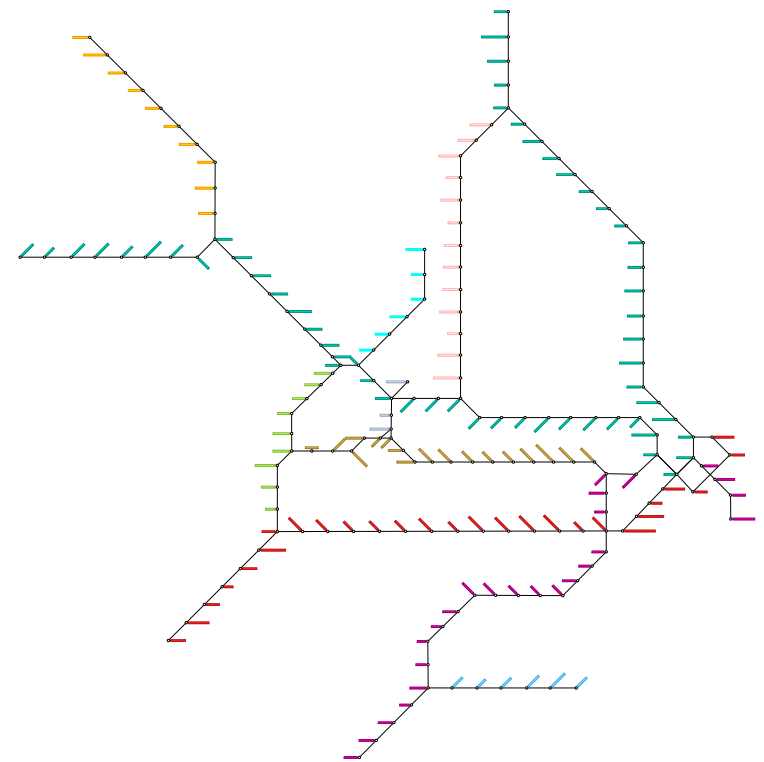

(b) GreedyAlg

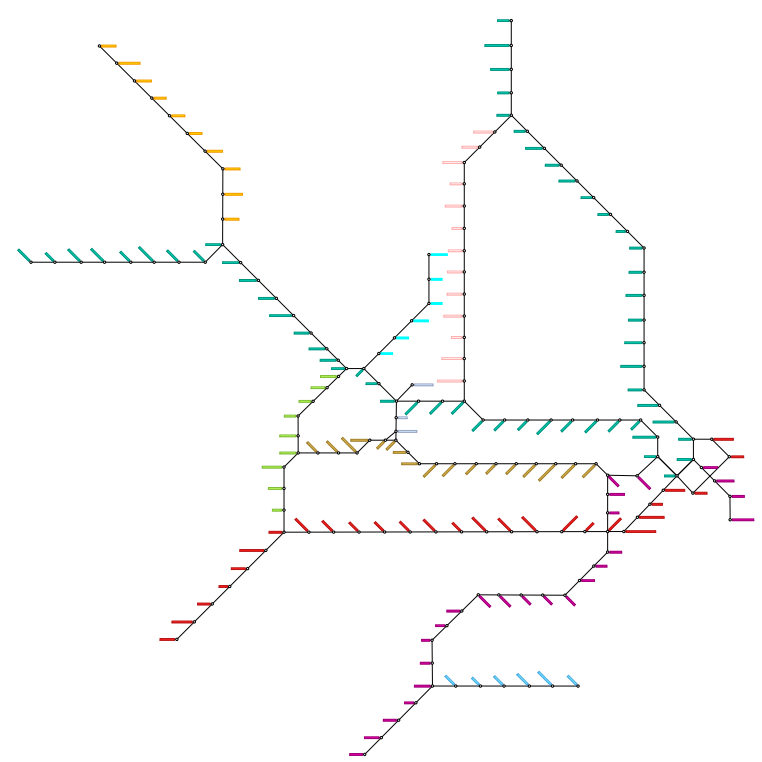

(d) ILPALG

Fig. 16: Labelings for instance Sydney3. 


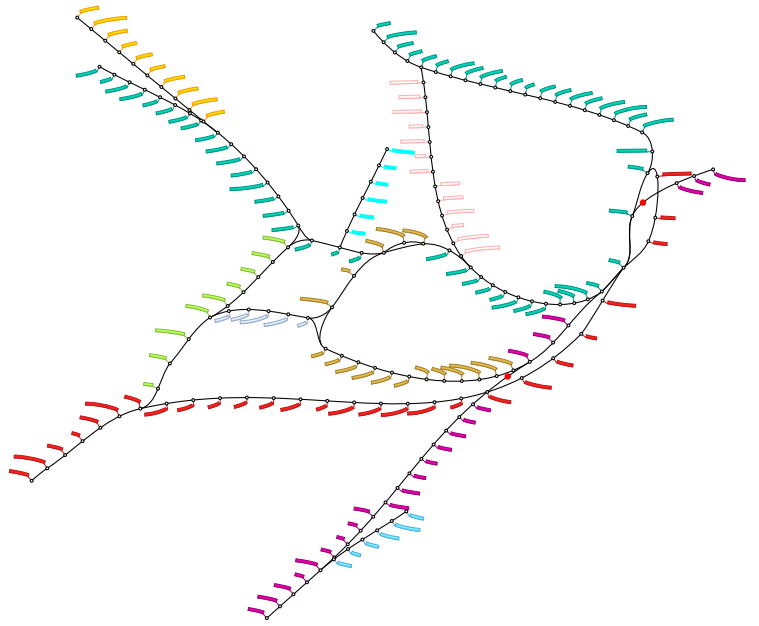

(a) DpAlg

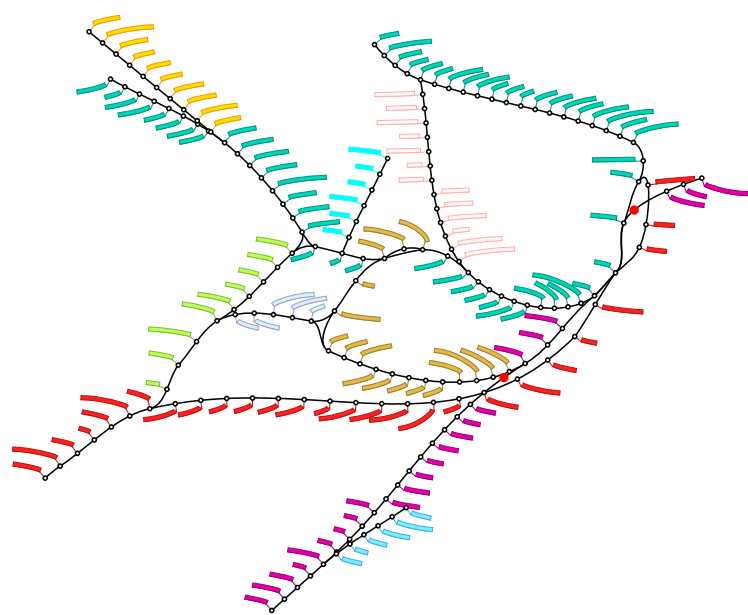

(c) ScaleAlg

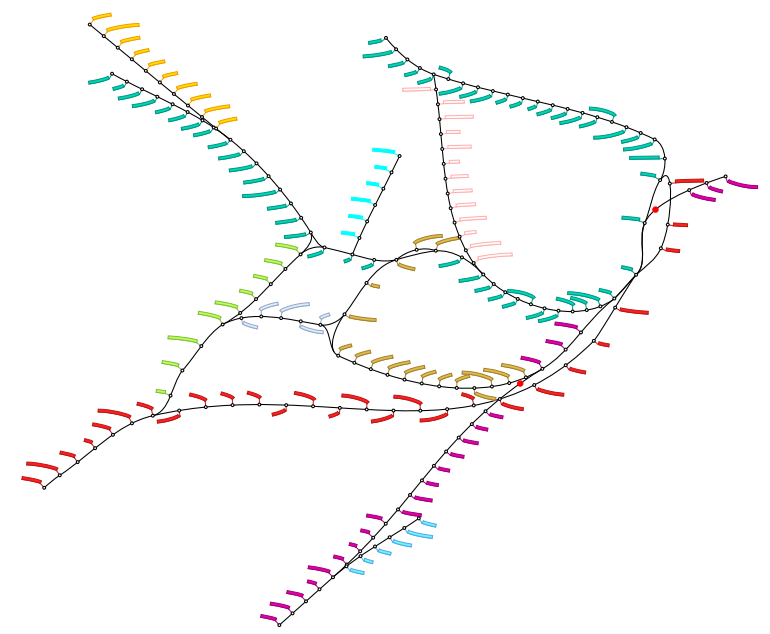

(b) GreedyAlg

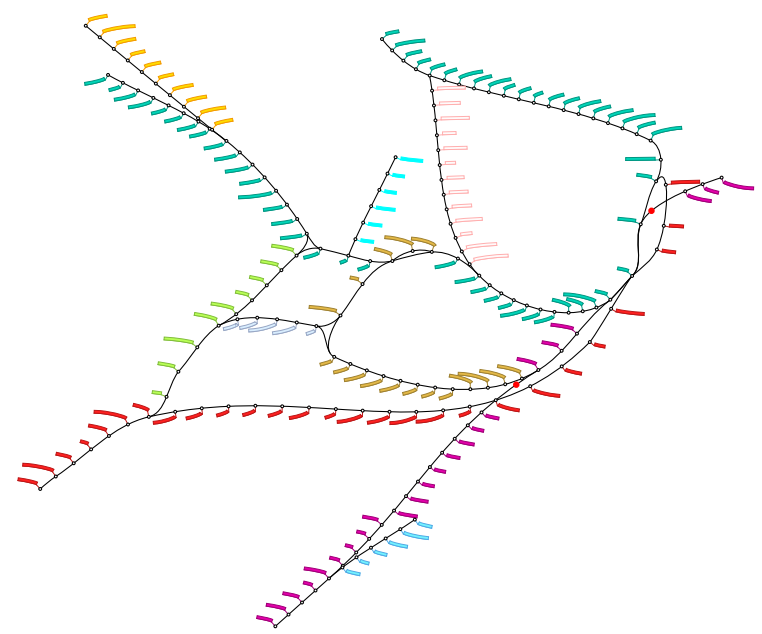

(d) IlpAlg

Fig. 17: Labelings for instance Sydney4. 


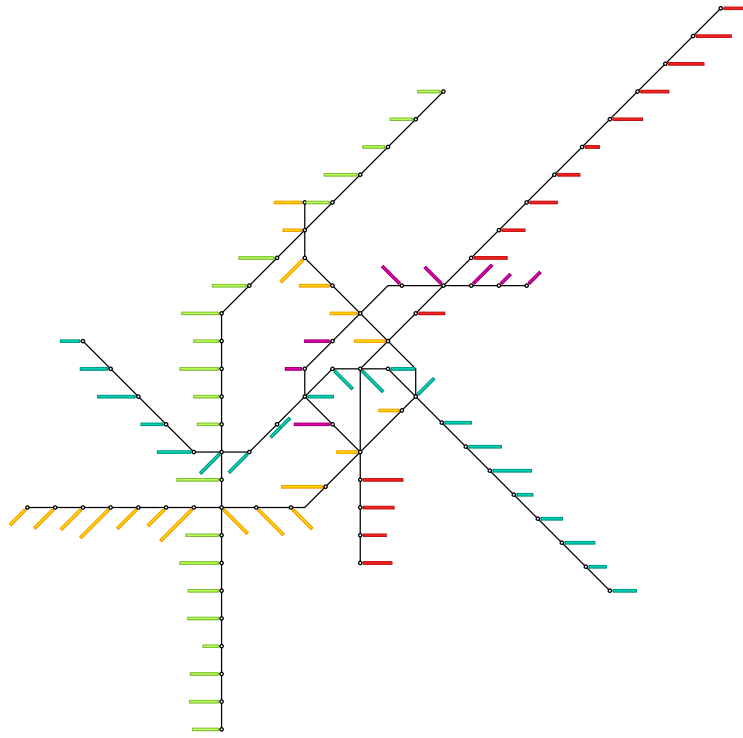

(a) DpAlg

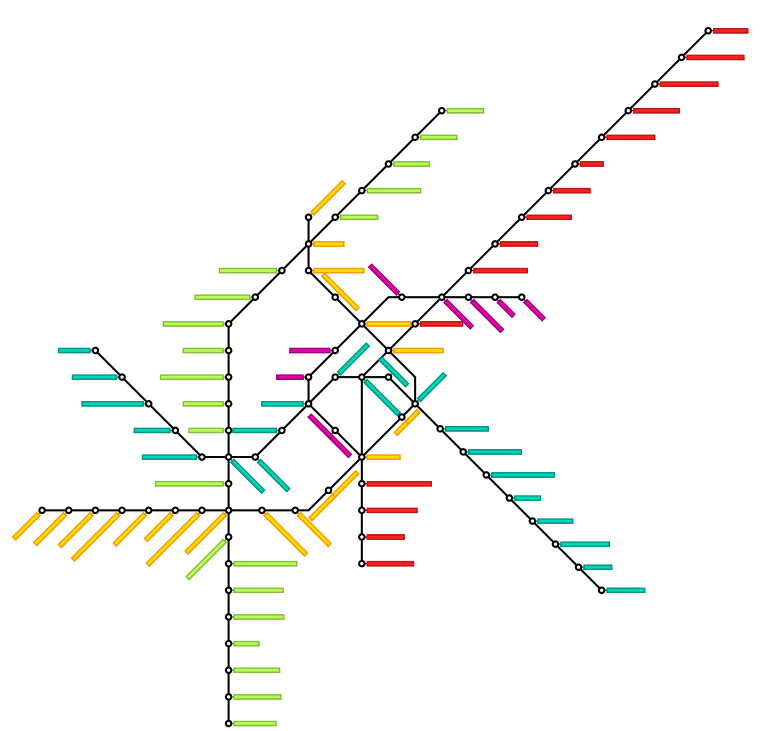

(c) ScaleAlg

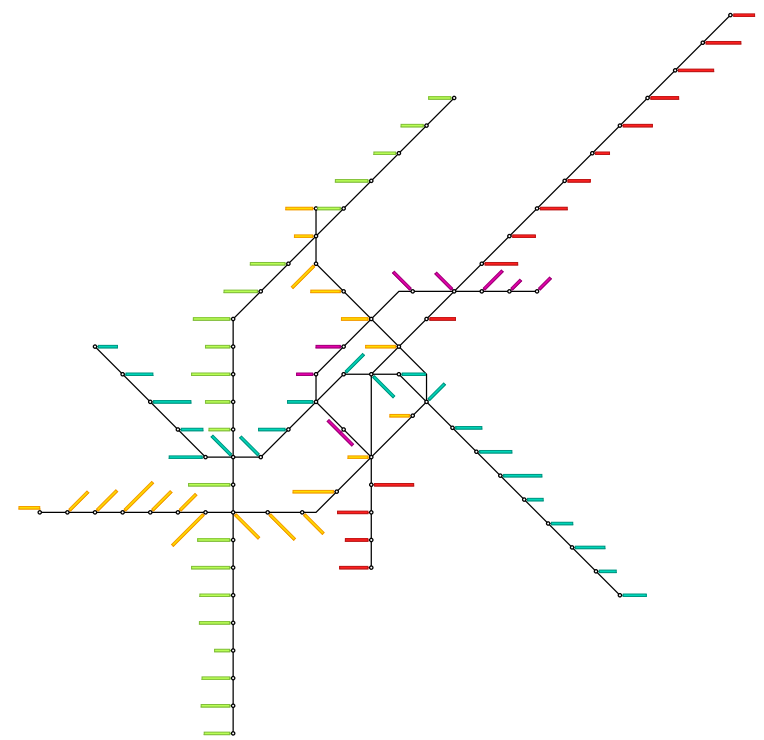

(b) GreedyAlG

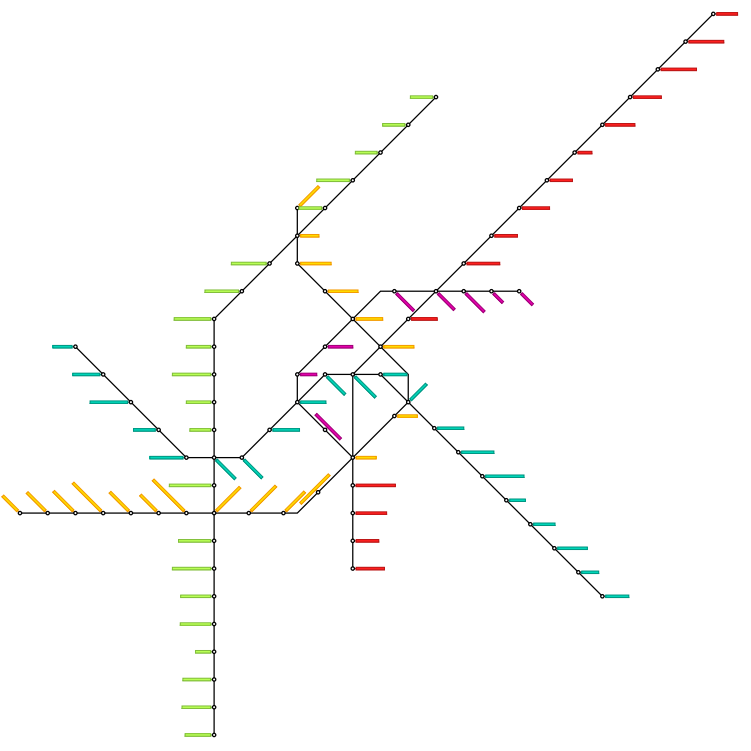

(d) IlpAlg

Fig. 18: Labelings for instance Vienna1. 


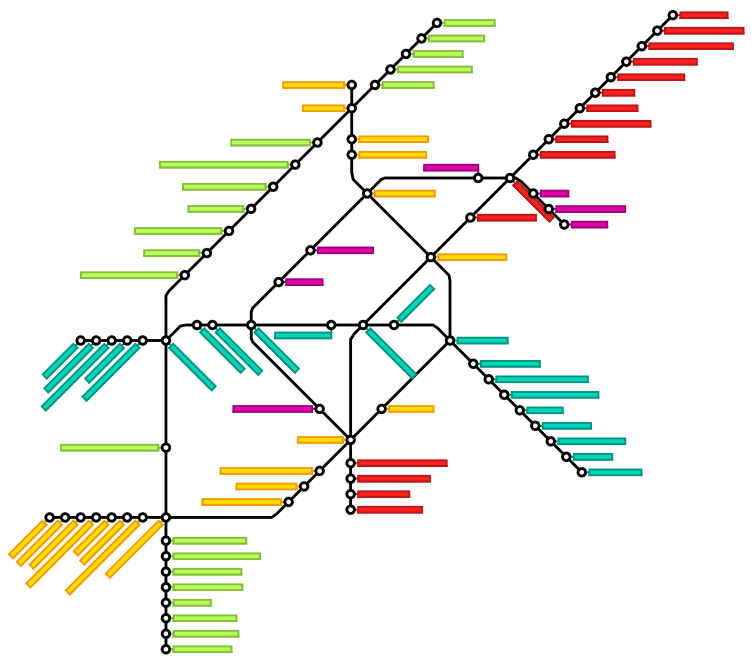

(a) DpAlg

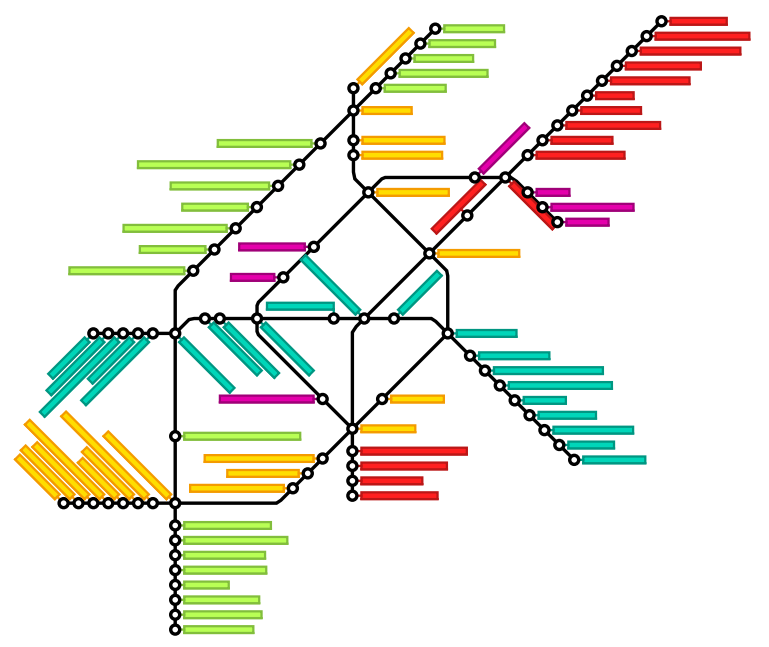

(c) ScAleAlg

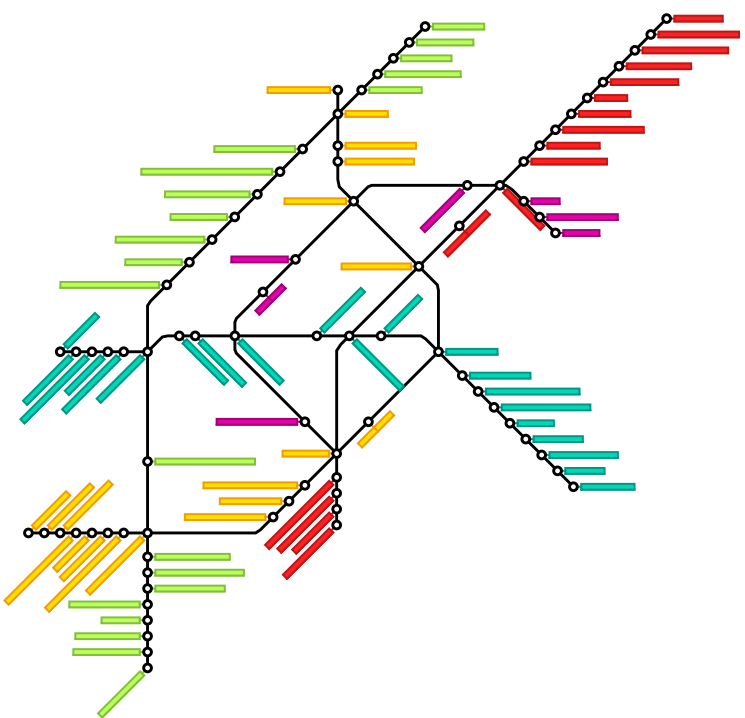

(b) GreedyAlg

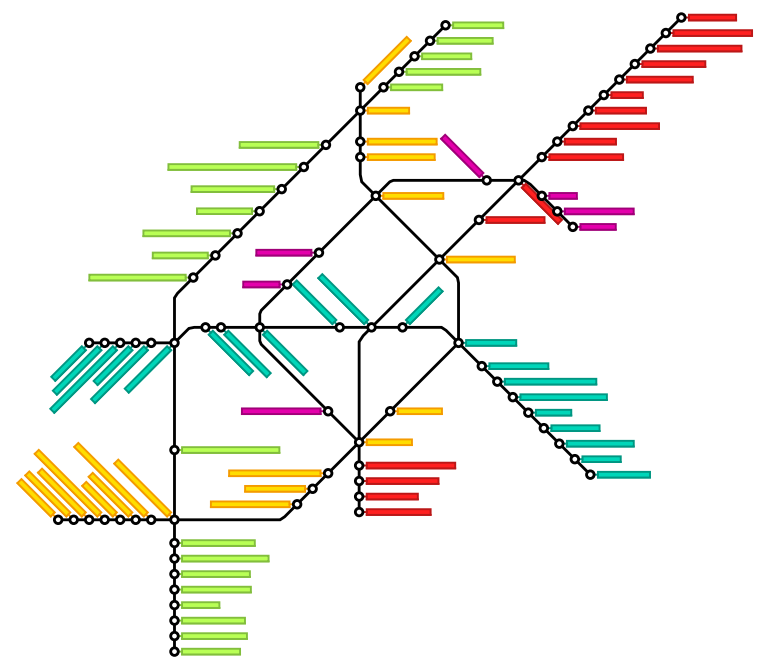

(d) ILPAlG

Fig. 19: Labelings for instance Vienna2. 


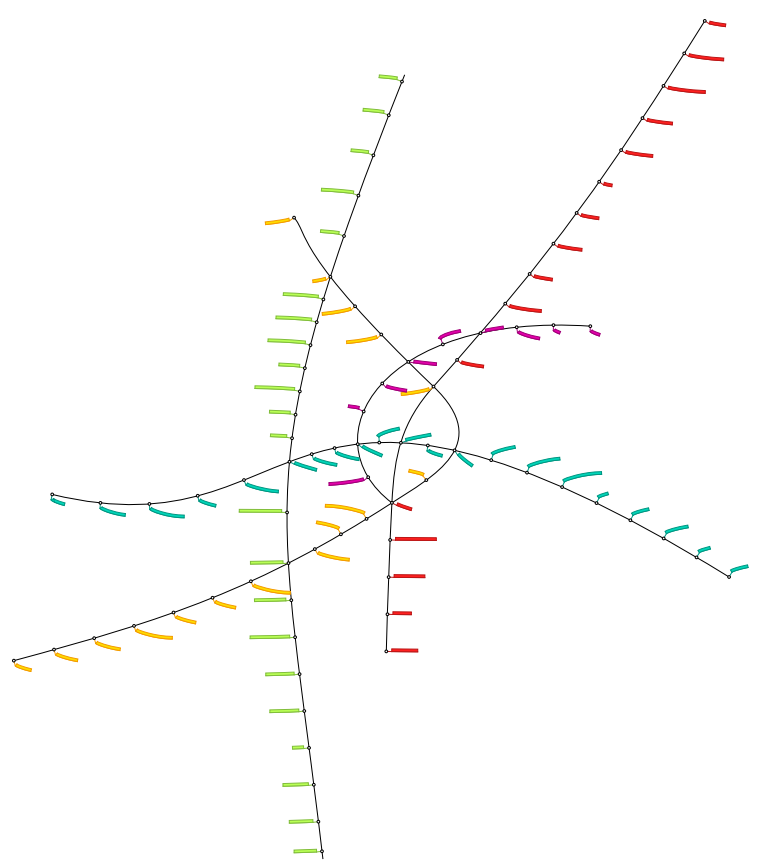

(a) DpAlg

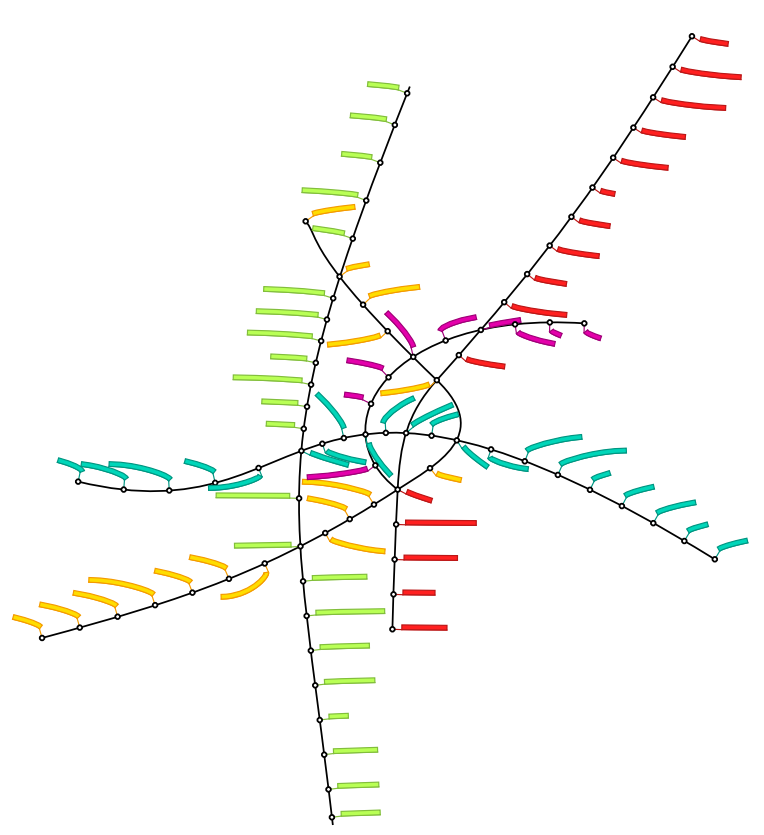

(c) ScaleAlg

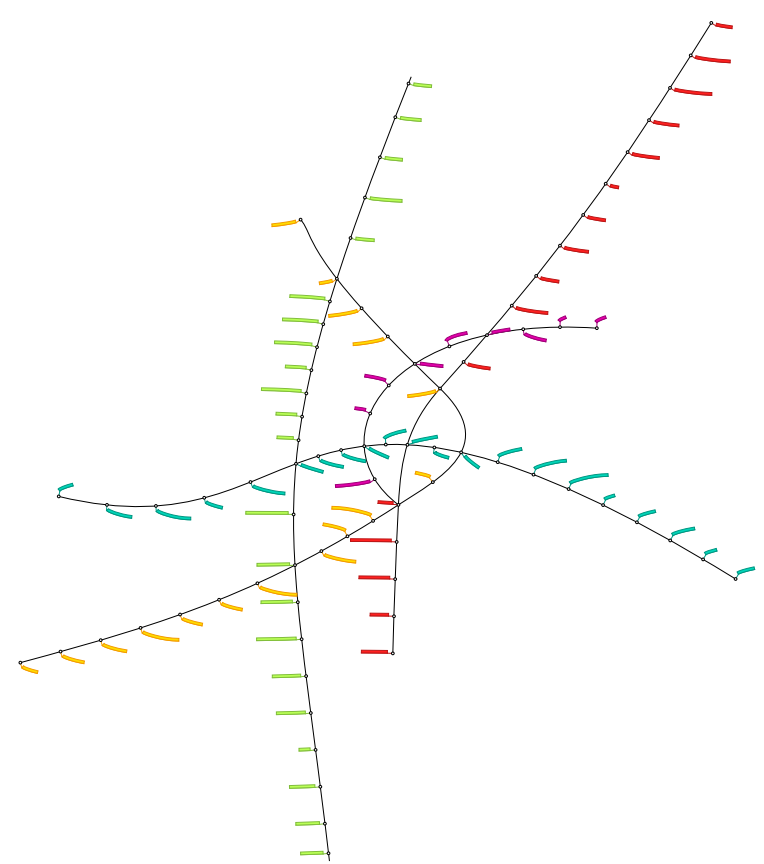

(b) GreedyAlg

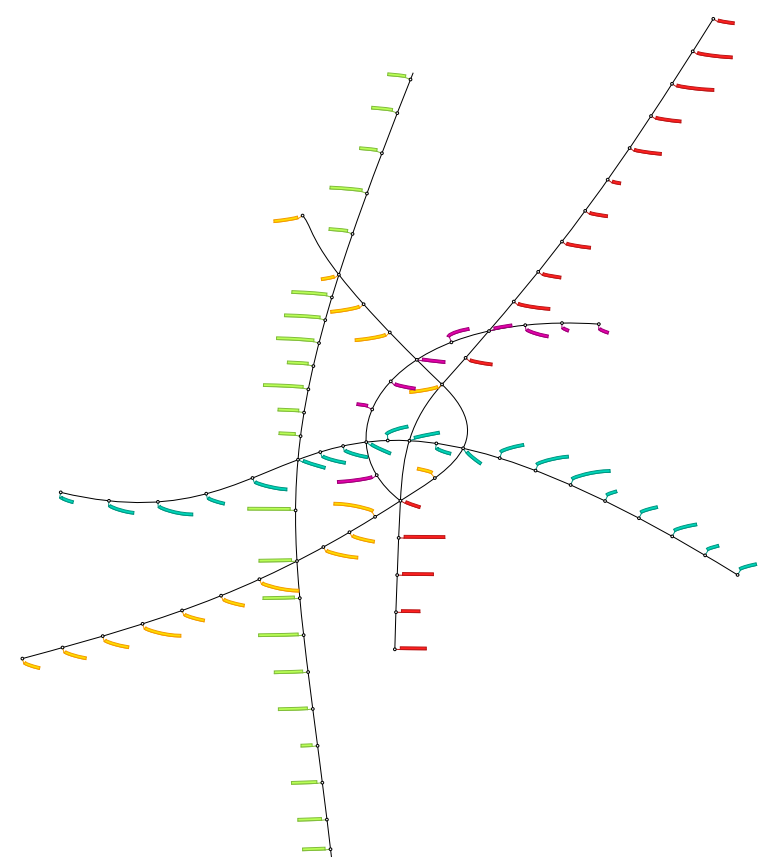

(d) IlpAlg

Fig. 20: Labelings for instance Vienna3. 